\title{
The 3-colored Ramsey Number of Odd Cycles
}

\author{
Yoshiharu Kohayakawa *† Miklós Simonovits ${ }^{\ddagger}$ Jozef Skokan $\mathbb{\|}$ \\ CDAM Research Report LSE-CDAM-2008-16
}

\begin{abstract}
Denote by $R(L, L, L)$ the minimum integer $N$ such that any 3 -coloring of the edges of the complete graph $K_{N}$ contains a monochromatic copy of a graph $L$. Bondy and Erdős conjectured that for an odd cycle on $n$ vertices $C_{n}$,

$$
R\left(C_{n}, C_{n}, C_{n}\right)=4 n-3 \text { for } n>3 .
$$

This is sharp if true.

Luczak proved that if $n$ is odd, then $R\left(C_{n}, C_{n}, C_{n}\right)=4 n+o(n)$, as $n \rightarrow \infty$. We prove here the exact Bondy-Erdös conjecture for sufficiently large values of $n$. We also describe the Ramsey-extremal colorings and prove some related stability theorems.

*Instituto de Matemática e Estatística, Universidade de São Paulo, Rua do Matão 1010, 05508090 São Paulo, Brazil, e-mail: yoshi@ime.usp.br

${ }^{\dagger}$ The author was partially supported by FAPESP and CNPq through a Temático-ProNEx project (Proc. FAPESP 2003/09925-5) and by CNPq (Proc. 306334/2004-6 and 479882/2004-5).

${ }_{\ddagger}^{\ddagger}$ Alfréd Rényi Institute of Mathematics, Hungarian Academy of Sciences H-1053 Budapest, Reáltanoda u. 13-15., Hungary, e-mail: miki@renyi.hu

$\S$ The author was partially supported by the Hungarian National Science Foundation grants OTKA T 026069, T 038210, and T 0234702, and by FAPESP (Proc. 04/02440-9).

`Department of Mathematics, London School of Economics, Houghton Street, London WC2A 2AE, United Kingdom and Department of Mathematics, MC-382, University of Illinois at UrbanaChampaign, 1409 W. Green Street, Urbana, IL 61801, USA, e-mail: jozef@member.ams.org

" The author was partially supported by the NSF grant INT-0305793, NSA grant H98230-04-10035, and by FAPESP (Proj. Temático-ProNEx Proc. FAPESP 2003/09925-5 and Proc. FAPESP
\end{abstract} 2004/15397-4). 


\section{Introduction}

For graphs $L_{1}, \ldots, L_{k}$, the Ramsey number $R\left(L_{1}, \ldots, L_{k}\right)$ is the minimum integer $N$ such that for any edge-coloring of the complete graph $K_{N}$ by $k$ colors there exists a color $i$ for which the corresponding color class contains $L_{i}$ as a subgraph.

The Ramsey number $R\left(C_{n}, C_{m}\right)$ has been studied by several authors, for example, Bondy and Erdős, [4], Faudree and Schelp, [8], Rosta, [22], and it is completely described and known that

$$
R\left(C_{n}, C_{n}\right)= \begin{cases}2 n-1, & \text { if } n \geq 5 \text { is odd, } \\ \frac{3 n}{2}-1, & \text { if } n \geq 6 \text { is even. }\end{cases}
$$

Bondy and Erdös [4] conjectured that if $n>3$ is odd, then

$$
R\left(C_{n}, C_{n}, C_{n}\right)=4 n-3 .
$$

This is sharp if true, as shown by Colorings 1 and 2 in Section 1.2. Euczak [20] proved that if $n$ is odd, then

$$
R\left(C_{n}, C_{n}, C_{n}\right)=4 n+o(n),
$$

as $n \rightarrow \infty$. In this paper we shall prove the existence of an $n_{0}$ for which the exact Bondy-Erdös conjecture holds if $n>n_{0}$. Our main result actually determines $R\left(C_{n_{1}}, C_{n_{2}}, C_{n_{3}}\right)$ for all odd and sufficiently large $n_{1}, n_{2}, n_{3}$.

Theorem 1. There exists an $n_{0}$ such that for all odd $n_{1}, n_{2}, n_{3}>n_{0}$, we have

$$
R\left(C_{n_{1}}, C_{n_{2}}, C_{n_{3}}\right)=4 \max \left\{n_{1}, n_{2}, n_{3}\right\}-3 .
$$

In particular, for $n>n_{0}$ odd,

$$
R\left(C_{n}, C_{n}, C_{n}\right)=4 n-3 .
$$

\subsection{Notation}

Our notation is quite standard. For graphs, the first subscripts mostly indicate the number of vertices, e.g., $G_{N}$ is always a graph of $N$ vertices, $C_{n}$ a cycle on $n$ vertices, $P_{\ell}$ is a path of $\ell$ vertices. We shall often "patch up" cycles from paths, and in these cases it is sometimes useful to specify the first and the last vertices of those paths. Hence, denote by $P(u, v)$ a path whose first vertex is $u$ and the last one is $v$, and we 
shall refer to it as a $(u, v)$-path. For non-empty disjoint sets $X$ and $Y$ we denote by $K(X, Y)$ the complete bipartite graph with bipartition $X \cup Y$.

For a graph $G$ we denote by $e(G)$ its number of edges. Given a set $X$ of vertices of $G, G[X]$ stands for the subgraph of $G$ induced by the vertices of $X . G \backslash X$ denotes the subgraph obtained by deleting the vertices of $X$ and the edges incident to $X$. We put $e(X):=e(G[X])$.

Given two disjoint sets of vertices, $X$ and $Y, E(X, Y)$ denotes the set of edges joining them, $e(X, Y):=|E(X, Y)|$, and $G[X, Y]$ is the bipartite subgraph of $G$ with bipartition $X \cup Y$ and edge set $E(X, Y)$. We also call

$$
d(X, Y):=\frac{e(X, Y)}{|X||Y|}
$$

the density of the pair $(X, Y)$ in $G$. When there is no danger of confusion, we use $u v \in G[X, Y]$ to mean $u \in X, v \in Y$, and $u v \in E(X, Y)$.

For a vertex $x$ we denote by $N(x)$ the set of all vertices adjacent to $x$ and set $\operatorname{deg}(x):=|N(x)|$ and $\operatorname{deg}(x, Y):=|N(x) \cap Y|$ (the degree of $x$ into $Y$ ). Similarly, for two vertices $x$ and $y$, we denote by $N(x, y)$ the set of vertices adjacent to both $x$ and $y$, and we set $\operatorname{deg}(x, y):=|N(x, y)|$.

Let $\delta(G)$ and $\Delta(G)$ denote the minimum and the maximum degree of $G$, respectively. We call $G$ t-complete if $\delta(G) \geq|V(G)|-1-t$. For a bipartite graph $G$ with bipartition $U \cup V$, we say that $G$ is $t$-complete if $\operatorname{deg}(u, V) \geq|V|-t$ for every $u \in U$ and $\operatorname{deg}(v, U) \geq|U|-t$ for every $v \in V$. We remark that the induced subgraphs of a $t$-complete graph are also $t$-complete.

Whenever we speak of colorings, we mean edge-colorings. Mostly we "use" three colors, red, blue and green, and the subgraphs of given colors will be indicated by superscripts: $G^{b}$ is the blue subgraph of $G, G^{r g}:=G^{r} \cup G^{g}$ is the red-green subgraph of $G$. However, the corresponding graph theoretical parameters (e.g., numbers of edges, degrees) will be indicated by subscripts: $e_{r}(X, Y)$ denotes the number of red edges between $X$ and $Y$ in an edge-colored graph, $\operatorname{deg}_{g}(x, Y)$ is the number of green edges joining $x$ to $Y$.

\subsection{Extremal colorings and stability}

Below we describe two colorings of $K_{4 m}$, providing the lower bound in Theorem 1 .

Coloring $1\left(\mathrm{EC}_{1}(m)\right)$. Take 4 sets $X_{1} \ldots, X_{4}$ of $m$ vertices each. Color the pairs inside each group by green, the edges of $K\left(X_{1}, X_{3}\right) \cup K\left(X_{2}, X_{4}\right)$ by red, $K\left(X_{1}, X_{2}\right) \cup$ 
$K\left(X_{3}, X_{4}\right)$ by blue, and the edges of $K\left(X_{1}, X_{4}\right) \cup K\left(X_{2}, X_{3}\right)$ arbitrarily, by red and blue.

The special feature of the second coloring is that it contains both blue and green complete graphs $K_{m}$.

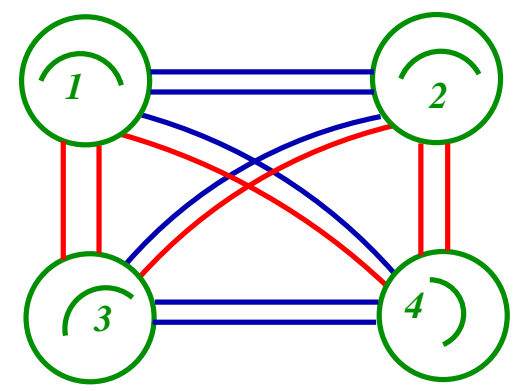

Fig 1: Coloring $\mathrm{EC}_{1}(m)$

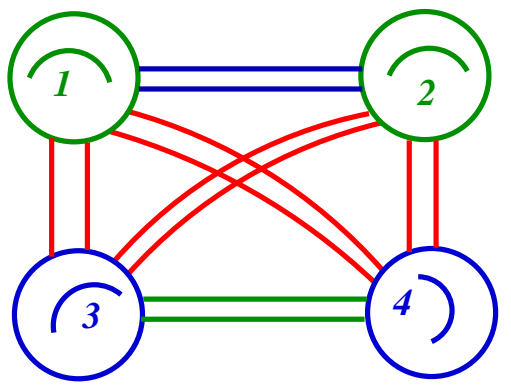

Fig 2: Coloring $\mathrm{EC}_{2}(m)$

Coloring $2\left(\mathrm{EC}_{2}(m)\right)$. Take 4 groups, $X_{1} \ldots, X_{4}$, of $m$ vertices each. Color all pairs in $X_{1}$ and $X_{2}$ by green, in $X_{3}$ and $X_{4}$ by blue. Then color $K\left(X_{3}, X_{4}\right)$ by green and $K\left(X_{1}, X_{2}\right)$ by blue. Finally, color the edges of $K\left(X_{1} \cup X_{2}, X_{3} \cup X_{4}\right)$ by red.

In the proof of the lower bound, we may assume that the color of the longest cycle is green, that is, $n:=n_{3}=\max n_{i}$.

Claim 2. For $n$ odd, colorings $\mathrm{EC}_{1}(n-1)$ and $\mathrm{EC}_{2}(n-1)$ do not contain monochromatic $C_{n}$. Moreover, $\mathrm{EC}_{1}(n-1)$ contains neither blue nor red odd cycles at all. Consequently, $R\left(C_{n_{1}}, C_{n_{2}}, C_{n_{3}}\right) \geq 4 \max \left\{n_{1}, n_{2}, n_{3}\right\}-3$.

Proof. In both colorings, each monochromatic subgraph is the vertex disjoint union of complete graphs $K_{n-1}$ and bipartite graphs (or just one of them). Hence these colorings do not contain green odd cycles of length $n=n_{3}$. In $\mathrm{EC}_{1}(n-1)$, both red and blue subgraphs are bipartite, thus $\mathrm{EC}_{1}(n-1)$ does not contain any odd red or blue cycles at all. $\left(\mathrm{EC}_{2}(n-1)\right.$ is an extremal coloring exactly if at least two of $n_{1}, n_{2}, n_{3}$ are maximal.)

We shall say that a 3-coloring of a graph $G$ contains $\mathrm{EC}_{1}(m)\left(\mathrm{EC}_{2}(m)\right.$, respectively) if there exists disjoint subsets $U_{1}, \ldots, U_{4}$ of $V(G)$, each of size $m$, and an injection $f: U_{i} \rightarrow X_{i}, i=1,2,3,4$, such that for every edge $x y$ in $G\left[U_{1} \cup \ldots \cup U_{4}\right]$, $x y$ has the same color in $G$ as $f(x) f(y)$ in $\mathrm{EC}_{1}(m)\left(\mathrm{EC}_{2}(m)\right.$, respectively).

We shall also say that a 3 -coloring of a graph $G$ can be embedded into coloring $\mathrm{EC}_{1}(m)\left(\mathrm{EC}_{2}(m)\right.$, respectively) if there exists an injection $f: V(G) \rightarrow X_{1} \cup X_{2} \cup$ 
$X_{3} \cup X_{4}$ such that for all edges $x y, v w \in E(G), x y$ and $v w$ have the same color in $G$ if and only if $f(x) f(y)$ and $f(v) f(w)$ have the same color in $\operatorname{EC}_{1}(m)\left(\mathrm{EC}_{2}(m)\right.$, respectively).

Our main result follows from Claim 2 combined with the following stability theorem.

Theorem 3. There exist constants $c>0$ and $N_{0} \in \mathbb{N}$ with the following property. For all odd integers $n_{1}, n_{2}, n_{3}>N_{0}$ set $n=\max \left\{n_{1}, n_{2}, n_{3}\right\}$ and let $N \geq(4-c) n$ be a natural number. Suppose that $K_{N}$ is 3-colored without red $C_{n_{1}}$, blue $C_{n_{2}}$, and green $C_{n_{3}}$.

Then $N<4 n-3$ and there is a subgraph $G$ of $K_{N}$ such that $e(G) \geq\left(\begin{array}{c}N \\ 2\end{array}\right)-10 N$ and the induced 3-coloring of $G$ can be embedded either into $\mathrm{EC}_{1}(n-1)$ or into $\mathrm{EC}_{2}(n-1)$.

Remark 4. The idea of using stability theorems in graph theory was initiated by the second author, back in the 60's and 70's, see, e.g., Simonovits [23], Erdös and Simonovits [7], Lovász and Simonovits [18, 19]. Since then this method has been successfully applied to many extremal problems, e.g., to find maximal triangle-free subgraphs of random graphs [1] or some recent exact hypergraph extremal theorems by Füredi and Simonovits [12], Keevash and Sudakov [14], and Füredi, Pikhurko and Simonovits $[10,11]$.

The proof of Theorem 3 comes in two stages: First we prove Theorem 5, a weakening of Theorem 3, where we have one additional condition that $G$ contains either $\mathrm{EC}_{1}(m)$ or $\mathrm{EC}_{2}(m)$, for some $m$ slightly larger than $n / 2$.

Theorem 5. Given three odd integers $n_{1}, n_{2}, n_{3} \geq 11$, set $n=\max \left\{n_{1}, n_{2}, n_{3}\right\}$. Let $N$ and $t$ be natural numbers for which $n>4 t+25$, and $N \geq 2 n+8 t+26$. Suppose that an $N$-vertex t-complete graph $G$ is 3-colored (in red, blue and green) without red $C_{n_{1}}$, blue $C_{n_{2}}$, and green $C_{n_{3}}$. Furthermore, suppose that there exists a t-complete subgraph $G^{\prime}$ of $G$ such that the induced 3-coloring of $G^{\prime}$ contains either $\mathrm{EC}_{1}\left(\frac{1}{2}(n+13)+2 t\right)$ or $\mathrm{EC}_{2}\left(\frac{1}{2}(n+13)+2 t\right)$.

Then $N<4 n-3$ and there exists a subgraph $G^{\prime \prime}$ of $G$ such that $e(G)-e\left(G^{\prime \prime}\right) \leq$ $10 N$ and the induced 3-coloring of $G^{\prime \prime}$ is embeddable either into $\mathrm{EC}_{1}(n-1)$ or into $\mathrm{EC}_{2}(n-1)$.

Then we shall prove that if a graph $G_{N}$ with large minimum degree is 3-colored and this 3-coloring has no long monochromatic cycles, then it must contain a relatively large colored subgraph of the structure described in Colorings 1 or 2. 
Theorem 6 (Ensuring The Additional Condition). There exists an $\eta_{6}>0$ such that for every $\eta \in\left(0, \eta_{6}\right)$ there is an $n_{6}=n_{6}(\eta)$ with the following property.

Let $n>n_{6}$ and $(4-\eta) n \leq N<(4+\eta) n$ be integers. Suppose that $G=G_{N}$ is a graph with $\delta(G) \geq\left(1-\eta^{4}\right) N$. Then for any 3 -coloring of the edges of $G$, if $G$ contains no monochromatic odd cycle longer than $(1+\eta / 100) n$, then one can find a subset $W^{*} \subset V(G)$ of $4\lceil(1 / 2+\eta / 4) n\rceil$ vertices such that the induced 3 -coloring of $G\left[W^{*}\right]$ can be embedded either into $\mathrm{EC}_{1}(\lceil(1 / 2+\eta / 4) n\rceil)$ or into $\mathrm{EC}_{2}(\lceil(1 / 2+\eta / 4) n\rceil)$.

We prove Theorem 3 in the following way: by combining Szemerédi's Regularity Lemma with Theorem 6, we find a large copy of Coloring 1 or 2 in a given 3-coloring of $K_{N}$ without red $C_{n_{1}}$, blue $C_{n_{2}}$, and green $C_{n_{3}}$. Then we apply Theorem 5 . The details of the proof can be found in Section 5 .

\section{Remark 7.}

(a) Since one of our main tools is the Szemerédi Regularity Lemma [24] (see Section 4), we cannot push down the threshold $n_{0}$ from which on our theorems hold (at least, not in this way).

(b) We do not really have to care for getting the exact length of a cycle: the Regularity Lemma enables us to get a monochromatic (odd) cycle of any length between a large constant and $m$ if we can find a monochromatic (odd) cycle longer than $m+\varepsilon m$. Mainly for this reason the proof of Theorem 3 reduces to the proof of Theorem 6 .

(c) Our proof of Theorem 1 has roots in the work of Euczak [20] into which we incorporated some new stability results.

The rest of this paper is organized as follows: In the next section we prove Theorem 5 and, in Section 3, we prove Theorem 6. Szemerédi's Regularity Lemma is given in Section 4. The details of the proof of Theorem 3 follow in Section 5.

\section{Proof of Theorem 5}

\subsection{Density statements}

We first list several results concerning cycles and paths in graphs with large minimum degree that we later use in the proof of Theorem 5 .

Theorem 8 (Erdős, Gallai, [5]). For any graph $G_{N}$ with

$$
e\left(G_{N}\right) \geq \frac{1}{2}(\ell-1)(N-1)+1,
$$


$G_{N}$ contains a cycle of length at least $\ell$.

When a graph has large minimum degree we can say a bit more. The following is a consequence of the well-known result of Bondy.

Theorem 9 (Bondy, [3]). Suppose that $G_{N}$ is a graph on $N$ vertices with $\delta\left(G_{N}\right)>$ $N / 2$. Then $C_{k} \subseteq G_{N}$ for each $k=3, \ldots, N$.

The next two lemmas provide sufficient conditions under which any two vertices of a given graph are connected by a path of prescribed length.

Lemma 10 (Williamson, [25]). Suppose that $G_{N}$ is a t-complete graph on $N$ vertices for some integer $1 \leq t \leq N / 2-2$. Then for every $2 \leq \ell \leq N-1$ and for any two vertices $u, v \in V\left(G_{N}\right)$ there is a $(u, v)$-path of length $\ell$ in $G_{N}$.

Lemma 11. Let $H$ be a t-complete bipartite graph with bipartition $U \cup V$. Then

(A) for any two vertices $u, u^{\prime} \in U$ there exists a $\left(u, u^{\prime}\right)$-path of length $2 \ell$ for every $1 \leq \ell \leq \min \{|U|-1,|V|-2 t\}$; the analogous statement, obtained by exchanging the two vertex classes, also holds.

(B) for any two vertices $u \in U, v \in V$ there exists a $(u, v)$-path of length $2 \ell+1$ for every odd $1 \leq \ell \leq \min \{|U|,|V|\}-2 t-1$;

(C) there is a cycle of length $2 \ell$ for every $2 \leq \ell \leq \min \{|U|,|V|\}-2 t$.

Proof. Without loss of generality, we may assume $|V|>2 t$. Fix any 2 vertices $u, u^{\prime} \in U$. Since $\operatorname{deg}\left(u, u^{\prime}, V\right) \geq|V|-2 t>0$, there exists a common neighbor $v \in V$ and, thus, a $\left(u, u^{\prime}\right)$-path of length 2 .

Suppose $u_{1}=u, v_{1}, u_{2}, v_{2}, \ldots, v_{\ell}, u_{\ell+1}=u^{\prime}$ is a $\left(u, u^{\prime}\right)$-path of length $2 \ell$ for some $\ell<\min \{|U|-1,|V|-2 t\}$. Since $\ell<|U|-1$, there is a vertex $w \in U \backslash$ $\left\{u_{1}, u_{2}, \ldots, u_{\ell+1}\right\}$. We have $\ell<|V|-2 t, \operatorname{deg}\left(w, u_{\ell+1}, V\right) \geq|V|-2 t, \operatorname{deg}\left(u_{\ell}, w, V\right) \geq$ $|V|-2 t$, and, therefore,

$\left|N\left(w, u_{\ell+1}\right) \backslash\left\{v_{1}, v_{2}, \ldots, v_{\ell-1}\right\}\right|,\left|N\left(u_{\ell}, w\right) \backslash\left\{v_{1}, v_{2}, \ldots, v_{\ell-1}\right\}\right| \geq|V|-2 t-(\ell-1) \geq 2$.

Hence, there exist two distinct neighbors, $v^{\prime}$ of $w, u_{\ell+1}$ and $v^{\prime \prime}$ of $u_{\ell}, w$, so that $u_{1}=u, v_{1}, u_{2}, v_{2}, \ldots, u_{\ell}, v^{\prime \prime}, w, v^{\prime}, u_{\ell+1}=u^{\prime}$ is a $\left(u, u^{\prime}\right)$-path of length $2 \ell+2$.

To see (B), for given $u \in U, v \in V$ we first find neighbors $u^{\prime} \neq u \in U$ of $v$ and $v^{\prime} \neq v \in V$ and then apply (A) to the $t$-complete bipartite graphs $H-v$ and $H-u$. Finally, (C) follows from (B) by taking any edge $u v$ of $H$. 


\subsection{Proof of Theorem 5}

Let the odd integers $n_{1}, n_{2}, n_{3} \geq 11$ be given and set $n=\max \left\{n_{1}, n_{2}, n_{3}\right\}$. Let $N$ and $t$ be natural numbers satisfying $t<\frac{1}{4}(n-25)$, and $N \geq 2 n+8 t+26$. Suppose that a $t$-complete graph $G=G_{N}$ is 3 -colored without red $C_{n_{1}}$, blue $C_{n_{2}}$, and green $C_{n_{3}}$.

Furthermore, suppose that there exists a $t$-complete subgraph $G^{\prime} \subset G$ whose induced 3-coloring contains either $\mathrm{EC}_{1}\left(\frac{1}{2}(n+13)+2 t\right)$ or $\mathrm{EC}_{2}\left(\frac{1}{2}(n+13)+2 t\right)$. Hence, there exist four disjoint sets of vertices $U_{1}, \ldots, U_{4},\left|U_{i}\right|=\frac{1}{2}(n+13)+2 t$, such that either

(a1) $G^{\prime}\left[U_{1}\right]$ and $G^{\prime}\left[U_{2}\right]$ are green, the other two, $G^{\prime}\left[U_{3}\right]$ and $G^{\prime}\left[U_{4}\right]$, are blue, and $G^{\prime}\left[U_{i}\right]$ is $t$-complete for $i=1,2,3,4$;

(a2) $G^{\prime}\left[U_{1}, U_{2}\right]$ is a blue $t$-complete bipartite graph, $G^{\prime}\left[U_{3}, U_{4}\right]$ is a green $t$-complete bipartite graph and $G^{\prime}\left[U_{1} \cup U_{2}, U_{3} \cup U_{4}\right]$ is a red $t$-complete bipartite graph;

or

(b1) $G^{\prime}\left[U_{1}\right], G^{\prime}\left[U_{2}\right], G^{\prime}\left[U_{3}\right]$ and $G^{\prime}\left[U_{4}\right]$ are green $t$-complete graphs;

(b2) $G^{\prime}\left[U_{1}, U_{2}\right]$ and $G^{\prime}\left[U_{3}, U_{4}\right]$ are blue $t$-complete bipartite graphs, $G^{\prime}\left[U_{1}, U_{3}\right]$ and $G^{\prime}\left[U_{2}, U_{4}\right]$ are red $t$-complete bipartite graphs, and $G^{\prime}\left[U_{1}, U_{4}\right]$ and $G^{\prime}\left[U_{2}, U_{3}\right]$ are $t$-complete bipartite graphs without green edges.

The $t$-completeness of $G^{\prime}\left[U_{i}\right]$ and $\left|U_{i}\right|=\frac{1}{2}(n+13)+2 t$ imply that $\delta\left(G^{\prime}\left[U_{i}\right]\right) \geq$ $\left|U_{i}\right|-1-t>\left|U_{i}\right| / 2$ for each $i=1,2,3,4$. By Theorem $9, G^{\prime}\left[U_{i}\right], i=1,2,3,4$, contains a cycle of every length between 3 and $\left|U_{i}\right|$. So we conclude that either

$$
n_{2}>\left|U_{1}\right|,\left|U_{2}\right| \text { and } n_{3}>\left|U_{3}\right|,\left|U_{4}\right|
$$

or

(b3) $n_{3}>\left|U_{i}\right|$ for $i=1,2,3,4$.

Let $W:=V(G) \backslash\left(U_{1} \cup U_{2} \cup U_{3} \cup U_{4}\right)$ be the set of "leftover" vertices in $V(G)$. We shall partition $W$ into 4 classes:

$$
W=U_{1}^{*} \cup U_{2}^{*} \cup U_{3}^{*} \cup U_{4}^{*}
$$

and show that coloring of $G_{N}$ induced on the union of classes $\tilde{U}_{i}:=U_{i} \cup U_{i}^{*}, i=$ $1,2,3,4$, is of the same type as the "special" coloring of $G^{\prime}$, apart from ten vertices.

More precisely, we shall prove the following: 
(A) for each $i=1,2,3,4$, all the edges in $U_{i}$ and all the edges between $U_{i}$ and $U_{i}^{*}$ have the same color as the edges in $G^{\prime}\left[U_{i}\right]$;

(B) For $i=1,2,3,4$, we have $\left|\tilde{U}_{i}\right|<n$. Thus, $N<4 n-3$;

(C) the sets $\tilde{U}_{i}, i=1,2,3,4$ determine either $\mathrm{EC}_{1}(n-1)$ or $\mathrm{EC}_{2}(n-1)$, with the exception of edges incident to at most 10 vertices.

Clearly, statements (A)-(C) prove the theorem.

We distinguish two cases depending on whether the conditions (a1)-(a3) or the conditions (b1)-(b3) apply.

\subsubsection{Case 1, corresponding to Coloring 1 .}

Here the conditions (b1)-(b3) apply. We first show that

all the edges of $G\left[U_{i}\right]$ have the same color as the edges of $G^{\prime}\left[U_{i}\right]$.

Let $u_{1} v_{1} \in G\left[U_{1}\right] \backslash G^{\prime}\left[U_{1}\right]$. By Lemma 11 and since $G^{\prime}\left[U_{1}, U_{2}\right]$ is a blue $t$-complete bipartite graph, there is a blue $\left(u_{1}, v_{1}\right)$-path of length $n_{2}-1$. Note that

$$
3 \leq n_{2}-1 \leq n-1<2\left(\frac{1}{2}(n+13)+2 t\right)-4 t-2 \leq 2 \min \left\{\left|U_{1}\right|-1,\left|U_{2}\right|-2 t\right\}
$$

and Lemma 11 applies. Similarly, since $G^{\prime}\left[U_{1}, U_{3}\right]$ is a red $t$-complete bipartite graph, there is a red $\left(u_{1}, v_{1}\right)$-path of length $n_{1}-1$. Thus, to avoid a red $C_{n_{1}}$ and blue $C_{n_{2}}$, $u_{1} v_{1}$ must be green. By symmetry, all the edges of $G\left[U_{i}\right], i=1,2,3,4$, are green.

Consider an arbitrary vertex $x \in W$. We claim that, for some $i=i(x), x$ is adjacent to all the vertices of $U_{i}$ in green. Suppose this is not the case. Then in red or blue, $x$ has at least one neighbor $u_{i}$ in each $U_{i}$. Since $G^{\prime}\left[U_{1}, U_{2}\right]$ is a blue $t$-complete bipartite graph, by Lemma 11 , it contains a blue path $P\left(u_{1}, u_{2}\right)$ of length $n_{2}-2$. Note that in this case, we have $3 \leq n_{2}-2 \leq n-2<2\left(\frac{1}{2}(n+13)+2 t\right)-4 t-1=$ $2\left(\min \left\{\left|U_{1}\right|,\left|U_{2}\right|\right\}-2 t-1\right)+1$.

Hence, at least one of $x u_{1}, x u_{2}$ must be red (otherwise we could find a blue $C_{n_{2}}$ using $x u_{1}, x u_{2}$ and $\left.P\left(u_{1}, u_{2}\right)\right)$. Assume that $x u_{1}$ is red. Then, since $G^{\prime}\left[U_{1}, U_{3}\right]$ is a red $t$-complete bipartite graph, $x u_{3}$ must be blue (by the same argument as above). The blue $t$-complete $G^{\prime}\left[U_{3}, U_{4}\right]$ implies that $x u_{4}$ is red, and the red $t$-complete $G^{\prime}\left[U_{2}, U_{4}\right]$ implies that $x u_{2}$ is blue.

Suppose there exists a blue edge $v_{1} v_{4}$ such that $v_{1} \in U_{1}$ and $v_{4} \in U_{4}$. By Lemma 11 and since $G^{\prime}\left[U_{1}, U_{2}\right]$ and $G^{\prime}\left[U_{3}, U_{4}\right]$ are blue $t$-complete bipartite graphs, 
there are a blue $\left(v_{1}, u_{2}\right)$-path of length $n_{2}-6$ and a blue $\left(u_{3}, v_{4}\right)$-path of length 3 . These two paths together with $x u_{2}, x u_{3}$, and $v_{1} v_{4}$ form a blue $C_{n_{2}}$, which is a contradiction. Therefore, $G\left[U_{1}, U_{4}\right]$ is red and $t$-complete. By Lemma 11 again, we find a red $\left(u_{1}, u_{4}\right)$-path of length $n_{1}-2$. This path forms a red $C_{n_{1}}$ with $u_{1} x$ and $x u_{4}-$ a contradiction.

Thus we can partition $W$ into $U_{1}^{*} \cup U_{2}^{*} \cup U_{3}^{*} \cup U_{4}^{*}$ by putting a vertex $w \in W$ into $U_{i}^{*}$ if all the edges in the set $E\left(w, U_{i}\right)$ are green. If there are two such $U_{i}$ 's, choose one of them arbitrarily. This completes the proof of (A).

To see (B), assume that one of the sets $\tilde{U}_{i}$ has at least $n \geq n_{3}$ vertices. In case of strict inequality, we omit some vertices from $U_{i}^{*}$ so that $\left|\tilde{U}_{i}\right|=\left|U_{i}\right|+\left|U_{i}^{*}\right|=n$. Recall that $\frac{1}{2}\left(n_{3}+3\right)<\frac{1}{2}(n+13)+2 t=\left|U_{i}\right|<n_{3}$ by (b3) and $6 \leq\left|U_{i}^{*}\right|=n-\left|U_{i}\right|=$ $\frac{1}{2}(n-13)-2 t$. Since $G^{g}\left[U_{i}, U_{i}^{*}\right]$ is $t$-complete, using Lemma 11 we find an $(u, v)$-path $P$ of length $\min \left\{2\left|U_{i}^{*}\right|, n_{3}-1\right\}$ for some green edge $u v, u, v \in U_{i}$. Note that in order to avoid a green $C_{n_{3}}$, we must have $2\left|U_{i}^{*}\right|<n_{3}-1$ and $P$ covers all the vertices of $U_{i}^{*}$. A simple counting shows that $G\left[\left(U_{i} \backslash P\right) \cup\{u, v\}\right]$ is a green $t$-complete graph satisfying $\left|\left(U_{i} \backslash P\right) \cup\{u, v\}\right|=\left|U_{i}\right|-\left|U_{i}^{*}\right|+1=14+4 t>2(t+2)$. Since

$$
2 \leq n_{3}-2\left|U_{i}^{*}\right| \leq n-2\left|U_{i}^{*}\right|=\left|U_{i}\right|-\left|U_{i}^{*}\right|=\left|\left(U_{i} \backslash P\right) \cup\{u, v\}\right|-1,
$$

by Lemma 10, $G\left[\left(U_{i} \backslash P\right) \cup\{u, v\}\right]$ contains a $(u, v)$-path $P^{\prime}$ of length $n_{3}-2\left|U_{i}^{*}\right|$. Clearly, $P \cup P^{\prime}$ is a green $C_{n_{3}}$.

From now on, we call two edges independent if they are vertex disjoint. In order to verify $(\mathrm{C})$, first we prove that

Claim 12. There are no two independent green edges between $\tilde{U}_{i}$ and $\tilde{U}_{j}$, for $i \neq j$.

Proof. Assume there are two green independent edges $e_{1}, e_{2}$ between $\tilde{U}_{1}$ and $\tilde{U}_{2}$. We claim that there exist distinct vertices $u_{1}, v_{1} \in U_{1}, u_{2}, v_{2} \in U_{2}$, a green path $P\left(u_{1}, u_{2}\right)$, and a green path $P\left(v_{1}, v_{2}\right)$, vertex-disjoint from the previous one, both of length at most 3 .

Indeed, we set $u_{i}=e_{1} \cap U_{i}$ if $e_{1} \cap U_{i}^{*}=\emptyset, i=1,2$. Otherwise, we define $u_{i}$ as any green neighbor of the vertex in $e_{1} \cap U_{i}^{*}$ in $U_{i}$. Similarly, we set $v_{i}=e_{2} \cap U_{i}$ if $e_{2} \cap U_{i}^{*}=\emptyset, i=1,2$, otherwise, $v_{i}$ is any green neighbor of the vertex in $e_{2} \cap U_{i}^{*}$ in $U_{i} \backslash\left\{u_{i}\right\}$. This is always possible because the bipartite graphs $G\left[U_{1}^{*}, U_{1}\right]$ and $G\left[U_{2}^{*}, U_{2}\right]$ are green and $t$-complete.

Since $G\left[U_{1}\right]$ and $G\left[U_{2}\right]$ are green and $t$-complete, there is a path $P\left(u_{i}, v_{i}\right) \subset G\left[U_{i}\right]$ of any length $\ell_{i}, 2 \leq \ell_{i} \leq\left|U_{i}\right|$, for $i=1,2$. Since $\left|U_{1}\right|+\left|U_{2}\right|>n+13+4 t>n_{3} \geq 11$, we 
can choose $2 \leq \ell_{1} \leq\left|U_{1}\right|$ and $2 \leq \ell_{2} \leq\left|U_{2}\right|$ so that $\ell_{1}+\ell_{2}+\left|P\left(u_{1}, u_{2}\right)\right|+\left|P\left(v_{1}, v_{2}\right)\right|=$ $n_{3}$. Clearly, $P\left(u_{1}, v_{1}\right), P\left(u_{2}, v_{2}\right)$ together with $P\left(u_{1}, u_{2}\right)$ and $P\left(v_{1}, v_{2}\right)$ form a green $C_{n_{3}}$ - a contradiction.

Hence there are no two independent green edges between $\tilde{U}_{1}$ and $\tilde{U}_{2}$ and, by symmetry, neither between any $\tilde{U}_{i}$ and any $\tilde{U}_{j}, i \neq j$. By removing the centers of at most 6 green stars we get that all $G\left[\tilde{U}_{i}, \tilde{U}_{j}\right]$ are $t$-complete and contain no green edges. Furthermore, we remove at most 3 vertices from each $\tilde{U}_{i}$.

Now we distinguish 4 sub-cases:

(1a) there exists either a red edge in one of $G\left[U_{1}, U_{2}\right] \backslash G^{\prime}\left[U_{1}, U_{2}\right], G\left[U_{3}, U_{4}\right] \backslash G^{\prime}\left[U_{3}, U_{4}\right]$ or a blue edge in one of $G\left[U_{1}, U_{3}\right] \backslash G^{\prime}\left[U_{1}, U_{3}\right], G\left[U_{2}, U_{4}\right] \backslash G^{\prime}\left[U_{2}, U_{4}\right]$;

(1b) $G\left[U_{1}, U_{2}\right], G\left[U_{3}, U_{4}\right]$ are blue, $G\left[U_{1}, U_{3}\right], G\left[U_{2}, U_{4}\right]$ are red, and there exists either a red edge in one of $G\left[U_{1}^{*}, U_{2}\right], G\left[U_{1}, U_{2}^{*}\right], G\left[U_{3}^{*}, U_{4}\right], G\left[U_{3}, U_{4}^{*}\right]$ or a blue edge in one of $G\left[U_{1}^{*}, U_{3}\right], G\left[U_{1}, U_{3}^{*}\right], G\left[U_{2}^{*}, U_{4}\right], G\left[U_{2}, U_{4}^{*}\right]$;

(1c) all the edges of $G\left[\tilde{U}_{1}, \tilde{U}_{2}\right] \backslash G\left[U_{1}^{*}, U_{2}^{*}\right]$ and $G\left[\tilde{U}_{3}, \tilde{U}_{4}\right] \backslash G\left[U_{3}^{*}, U_{4}^{*}\right]$ are blue, all the edges of $G\left[\tilde{U}_{1}, \tilde{U}_{3}\right] \backslash G\left[U_{1}^{*}, U_{3}^{*}\right]$ and $G\left[\tilde{U}_{2}, \tilde{U}_{4}\right] \backslash G\left[U_{2}^{*}, U_{4}^{*}\right]$ are red, and either one of $G\left[U_{1}^{*}, U_{2}^{*}\right], G\left[U_{3}^{*}, U_{4}^{*}\right]$ contains a red edge or one of $G\left[U_{1}^{*}, U_{3}^{*}\right], G\left[U_{2}^{*}, U_{4}^{*}\right]$ contains a blue edge;

(1d) all the edges of $G\left[\tilde{U}_{1}, \tilde{U}_{2}\right]$ and $G\left[\tilde{U}_{3}, \tilde{U}_{4}\right]$ are blue and all the edges of $G\left[\tilde{U}_{1}, \tilde{U}_{3}\right]$ and $G\left[\tilde{U}_{2}, \tilde{U}_{4}\right]$ are red.

We will show that sub-case (1a) reduces to (1b), (1c) or (1d), sub-case (1b) reduces to $(1 \mathrm{c})$ or $(1 \mathrm{~d})$, sub-case $(1 \mathrm{c})$ reduces to $(1 \mathrm{~d})$, and (1d) implies $(\mathrm{C})$. This will conclude the proof in the Case 1.

(1a) reduces to $(1 \mathrm{~b}),(1 \mathrm{c})$ or $(1 \mathrm{~d})$.

By symmetry, suppose that $v_{3} v_{4} \in G\left[U_{3}, U_{4}\right] \backslash G^{\prime}\left[U_{3}, U_{4}\right], v_{3} \in U_{3}, v_{4} \in U_{4}$, is red. We are going to show that all the edges of $G\left[U_{1}, U_{4}\right], G\left[U_{2}, U_{3}\right]$ are blue and all the edges of $G\left[U_{1}, U_{3}\right], G\left[U_{2}, U_{4}\right]$ are red. By renaming $U_{i}$ to $U_{i+1}$ and $U_{i}^{*}$ to $U_{i+1}^{*}$, we see that one of (1b), (1c), (1d) must hold. The assertion of this sub-case follows from the following two claims.

Claim 13. All the edges of $G\left[U_{1}, U_{4}\right]$ and $G\left[U_{2}, U_{3}\right]$ are blue.

Proof. To the contrary, let $w_{1} w_{4} \in G\left[U_{1}, U_{4}\right]$ be a red edge. By Lemma 11, there is a path $P\left(v_{4}, w_{4}\right)$ of length 2 (or 0 if $\left.v_{4}=w_{4}\right)$ in the red $t$-complete $G^{\prime}\left[U_{3}, U_{4}\right]$. Since 
$G^{\prime}\left[U_{1}, U_{3}\right]$ is also red and $t$-complete, the same lemma implies the existence of a red path $P\left(w_{1}, v_{3}\right)$ of length $n_{1}-2-\left|P\left(v_{4}, w_{4}\right)\right|$. Clearly, $P\left(v_{4}, w_{4}\right), P\left(w_{1}, v_{3}\right), v_{3} v_{4}$, and $w_{1} w_{4}$ form a red cycle of length $n_{1}$ - a contradiction. Similarly, $G\left[U_{2}, U_{3}\right]$ is blue.

Claim 14. All the edges of $G\left[U_{1}, U_{3}\right]$ and $G\left[U_{2}, U_{4}\right]$ are red.

Proof. To the contrary, suppose that $w_{2} w_{4} \in G\left[U_{2}, U_{4}\right]$ is a blue edge. Since $G^{\prime}\left[U_{3}, U_{4}\right]$ is blue and $t$-complete, there exists a blue edge $w_{3} w_{4}$ for some $w_{3} \in U_{3}$. By the previous claim, $G\left[U_{2}, U_{3}\right]$ is a blue $t$-complete bipartite graph, hence, by Lemma 11, there is a $\left(w_{2}, w_{3}\right)$-path $P$ of length $n_{2}-2$. Clearly, $P, w_{2} w_{3}$, and $w_{3} w_{4}$ form a blue cycle of length $n_{2}$ - a contradiction. For $G\left[U_{1}, U_{3}\right]$ we proceed similarly.

(1b) reduces to $(1 \mathrm{c})$ or $(\mathbf{1 d})$. Without loss of generality we may assume that there exists a red edge $u_{1} u_{2}$ with $u_{1} \in U_{1}^{*}$ and $u_{2} \in U_{2}$. We are going to show that all the edges of $G\left[\tilde{U}_{1}, \tilde{U}_{3}\right] \backslash G\left[U_{1}^{*}, U_{3}^{*}\right]$ and $G\left[\tilde{U}_{2}, \tilde{U}_{4}\right] \backslash G\left[U_{2}^{*}, U_{4}^{*}\right]$ are red, and all the edges of $G\left[\tilde{U}_{2}, \tilde{U}_{3}\right] \backslash G\left[U_{2}^{*}, U_{3}^{*}\right]$ and $G\left[\tilde{U}_{1}, \tilde{U}_{4}\right] \backslash G\left[U_{1}^{*}, U_{4}^{*}\right]$ are blue. By renaming $U_{i}$ to $U_{i+1}$ and $U_{i}^{*}$ to $U_{i+1}^{*}$, we see that either (1c) or (1d) must hold.

Since $G\left[U_{2}, U_{4}\right]$ is red and $t$-complete, every vertex in $U_{2}$ is connected to each vertex in $U_{4}$ by a red path of length $n_{1}-2$, by Lemma 11 . To avoid a red $C_{n_{1}}$, all the edges from $u_{1}$ to $U_{4}$ are blue. Similarly, every vertex in $U_{4}$ is connected to any vertex in $U_{3}$ by a blue path of length $n_{2}-2$. To avoid a blue $C_{n_{2}}$, all the edges from $u_{1}$ to $U_{3}$ must be red.

We claim that all the edges of $G\left[U_{1}, U_{4}\right]$ are red. Indeed, let $w_{1} w_{4} \in G\left[U_{1}, U_{4}\right]$ be a red edge and let $w_{3} \in U_{3}$ be any common neighbor of $u_{1}$ and $w_{1}$. Since $G\left[U_{2}, U_{4}\right]$ is red and $t$-complete, there is a red $\left(w_{4}, u_{2}\right)$-path of length $n_{1}-4$. This path together with red edges $w_{1} w_{4}, u_{1} u_{2}, u_{1} w_{3}, w_{3} w_{1}$ form a red $C_{n_{1}}-$ a contradiction again. So $G\left[U_{1}, U_{4}\right]$ is completely blue. Similarly, all the edges of $G\left[U_{2}, U_{3}\right]$ are blue.

The assertion of this sub-case follows from the following two claims.

Claim 15. All the edges of $G\left[U_{1}, U_{3}^{*}\right], G\left[U_{1}^{*}, U_{3}\right], G\left[U_{2}, U_{4}^{*}\right]$ and $G\left[U_{2}^{*}, U_{4}\right]$ are red.

Proof. Suppose that $w_{2} w_{4} \in G\left[U_{2}^{*}, U_{4}\right], w_{2} \in U_{2}^{*}, w_{4} \in U_{4}$, is a blue edge and consider arbitrary neighbors $w_{1} \in U_{1}, w_{3} \in U_{3}$ of $w_{2}$. If either $w_{1} w_{2}$ or $w_{2} w_{3}$ is blue, then we have a blue $C_{n_{2}}$ because we can join $w_{1}$ and $w_{4}$ by a blue path of length $n_{2}-2$ in $G\left[U_{1}, U_{4}\right]$ and $w_{3}$ and $w_{4}$ by a blue path of length $n_{2}-2$ in $G\left[U_{3}, U_{4}\right]$. (Both graph are $t$-complete and Lemma 11 applies.) Hence $w_{1} w_{2}$ and $w_{2} w_{3}$ are both red 
and we obtain a red $C_{n_{1}}$ by finding a red $\left(w_{1}, w_{3}\right)$-path of length $n_{1}-2$ in the red $t$-complete graph $G\left[U_{1}, U_{3}\right]$. We would proceed similarly if there were any blue edges in $G\left[U_{1}, U_{3}^{*}\right], G\left[U_{1}^{*}, U_{3}\right]$, or $G\left[U_{2}, U_{4}^{*}\right]$.

Claim 16. All the edges of $G\left[U_{1}, U_{4}^{*}\right], G\left[U_{1}^{*}, U_{4}\right], G\left[U_{2}, U_{3}^{*}\right]$, and $G\left[U_{2}^{*}, U_{3}\right]$ are blue.

Proof. To the contrary, let $v_{1} v_{4} \in G\left[U_{1}, U_{4}^{*}\right]$ be any red edge. By (b2) and Claim 15, $G\left[\tilde{U}_{1}, U_{3}\right]$ is red and $t$-complete, thus, by Lemma 11 , there is an $\left(u_{1}, v_{1}\right)$-path of length $n_{1}-5$. Similarly, since $G\left[U_{2}, \tilde{U}_{4}\right]$ is red and $t$-complete, there exists a red $\left(u_{2}, v_{4}\right)$-path of length 3 . Clearly, these two red paths and $u_{1} u_{2}, v_{1} v_{3}$ form a red $C_{n_{1}}$ - a contradiction. We would proceed in the same way if there were any red edges in $G\left[U_{1}^{*}, U_{4}\right], G\left[U_{2}, U_{3}^{*}\right]$, or $G\left[U_{2}^{*}, U_{4}\right]$.

(1c) reduces to (1d). One of our assumptions in this sub-case is that either $G\left[U_{1}^{*}, U_{2}^{*}\right] \cup G\left[U_{3}^{*}, U_{4}^{*}\right]$ contains a red edge or $G\left[U_{1}^{*}, U_{3}^{*}\right] \cup G\left[U_{2}^{*}, U_{4}^{*}\right]$ contains a blue edge. Without loss of generality we may assume that

$$
\text { there exists a red edge } u_{1} u_{2} \in G\left[U_{1}^{*}, U_{2}^{*}\right] \text {. }
$$

In Claims 17 and 18 below we are going to prove that all the edges of $G\left[\tilde{U}_{1}, \tilde{U}_{3}\right]$ and $G\left[\tilde{U}_{2}, \tilde{U}_{4}\right]$ are red and all the edges of $G\left[\tilde{U}_{1}, \tilde{U}_{4}\right]$ and $G\left[\tilde{U}_{2}, \tilde{U}_{3}\right]$ are blue. However, now we are in the sub-case (1d) with the rôle of $U_{i}$ and $U_{i}^{*}$ played by $U_{i+1}$ and $U_{i+1}^{*}$.

Claim 17. All the edges of $G\left[\tilde{U}_{1}, \tilde{U}_{4}\right]$ and $G\left[\tilde{U}_{2}, \tilde{U}_{3}\right]$ are blue.

Proof. Indeed, suppose that some $v_{1} v_{4} \in G\left[\tilde{U}_{1}, \tilde{U}_{4}\right]$ is red. Since $G\left[\tilde{U}_{1}, U_{3}\right]$ is red and $t$-complete, by Lemma 11 there is a $\left(u_{1}, v_{1}\right)$-path $P$ of length 2 . (If $u_{1}=v_{1}$, we set $P=u_{1}$.) Both $G\left[\tilde{U}_{2}, U_{4}\right]$ and $G\left[U_{2}, \tilde{U}_{4}\right]$ are red $t$-complete bipartite graphs with at least $\frac{1}{2}(n+13)+2 t-3$ vertices in each partite set. By Lemma 11 there exists a red $\left(u_{2}, v_{4}\right)$-path $P^{\prime}$ of length $n_{1}-2-|P|$ in $G\left[\tilde{U}_{2}, U_{4}\right]$ because

$$
\begin{aligned}
n_{1}-2-|P| & \leq 2\left(\frac{1}{2}(n+13)+2 t-3\right)-4 t-1 \\
& \leq 2\left(\max \left\{\left|\tilde{U}_{2}\right|,\left|U_{4}\right|\right\}-2 t-1\right)+1 .
\end{aligned}
$$

Clearly, $P, P^{\prime}, u_{1} u_{2}, v_{1} v_{4}$ form a red $C_{n_{1}}$ - a contradiction. We proceed in the same way for any red edge in $G\left[\tilde{U}_{2}, \tilde{U}_{3}\right]$. 
Claim 18. All the edges of $G\left[\tilde{U}_{1}, \tilde{U}_{3}\right]$ and $G\left[\tilde{U}_{2}, \tilde{U}_{4}\right]$ are red.

Proof. By the assumptions of (1c), we know that all the edges of $G\left[\tilde{U}_{1}, \tilde{U}_{3}\right] \backslash G\left[U_{1}^{*}, U_{3}^{*}\right]$ and $G\left[\tilde{U}_{2}, \tilde{U}_{4}\right] \backslash G\left[U_{2}^{*}, U_{4}^{*}\right]$ are red. Hence what remains to verify is that all the edges of $G\left[U_{1}^{*}, U_{3}^{*}\right]$ and $G\left[U_{2}^{*}, U_{4}^{*}\right]$ are red.

To the contrary, let $u_{2} u_{4} \in G\left[U_{2}^{*}, U_{4}^{*}\right]$ be a blue edge. Since $G\left[U_{1}, \tilde{U}_{2}\right]$ and $G\left[\tilde{U}_{1}, \tilde{U}_{4}\right]$ are blue and $t$-complete (by the assumptions of (1c) and Claim 17), there are two distinct vertices $w_{1}, w_{1}^{\prime} \in U_{1}$ such that $u_{2} w_{1}$ and $u_{4} w_{1}^{\prime}$ are blue. By Lemma 11, $w_{1}$ and $w_{1}^{\prime}$ are joined by a blue path of length $n_{2}-3$ in the blue $t$-complete graph $G\left[U_{1}, U_{2}\right]$. This path together with $u_{2} u_{4}, u_{2} w_{1}$ and $u_{4} w_{1}^{\prime}$ form a blue $C_{n_{2}}$ - a contradiction. We can rule out the existence of any blue edge in $G\left[U_{1}^{*}, U_{3}^{*}\right]$ similarly.

(1d) implies (C). We know that all the edges of $G\left[\tilde{U}_{1}, \tilde{U}_{2}\right]$ and $G\left[\tilde{U}_{3}, \tilde{U}_{4}\right]$ are blue, all the edges of $G\left[\tilde{U}_{1}, \tilde{U}_{3}\right]$ and $G\left[\tilde{U}_{2}, \tilde{U}_{4}\right]$ are red, and all 4 graphs are $t$-complete. For every $i=1,2,3,4$, we also have $\left|\tilde{U}_{i}\right| \geq \frac{1}{2}(n+13)+2 t-3>\max \left\{\frac{1}{2}\left(n_{1}-1\right)+2 t+1\right.$, $\left.\frac{1}{2}\left(n_{2}-1\right)+2 t+1\right\}$. Therefore, Lemma 11 implies that any two vertices of $U_{i}^{*}$ are connected by a red path of length $n_{1}-1$ and by a blue path of length $n_{2}-1$. Hence all the edges within $U_{i}^{*}$ are green and $(\mathrm{C})$ holds. The second part of $(\mathrm{C})$ follows from the fact that in the above argument we ignored only edges incident to at most six vertices.

\subsubsection{Case 2, corresponding to Coloring 2.}

This is the easier case, since here the coloring is "more" unique. Now the conditions (a1)-(a3) apply. First of all, similarly to (4), all the edges of $G\left[U_{i}\right]$ must have the same color as the edges of $G^{\prime}\left[U_{i}\right]$.

Secondly, since $G^{\prime}\left[U_{1} \cup U_{2}, U_{3} \cup U_{4}\right]$ is a red $t$-complete bipartite graph and $\mid U_{1} \cup$ $U_{2}|,| U_{3} \cup U_{4} \mid=n+13+4 t>\frac{1}{2}\left(n_{1}-3\right)+2 t+1$, Lemma 11 implies that every vertex of $U_{1} \cup U_{2}$ can be connected to any vertex of $U_{3} \cup U_{4}$ by a red path of length $n_{1}-2$. Hence, no vertex of $W$ can be adjacent to both $U_{1} \cup U_{2}$ and $U_{3} \cup U_{4}$ by a red edge, because that would imply having a red cycle of length $n_{1}$. Thus the set $W$ splits into two disjoint subsets $W_{12}$ and $W_{34}$ so that the vertices of $W_{12}$ are adjacent to $U_{1} \cup U_{2}$ only in blue and green and the vertices of $W_{34}$ are adjacent to $U_{3} \cup U_{4}$ only in blue and green (again, ties are decided arbitrarily).

Since $G^{\prime}\left[U_{1}, U_{2}\right]$ is blue and it is a $t$-complete bipartite graph, by Lemma 11 , for each $u_{1} \in U_{1}$ and $u_{2} \in U_{2}$, it contains a blue $\left(u_{1}, u_{2}\right)$-path of length $n_{2}-2$. Hence 
no vertex of $W_{12}$ can be adjacent to both $U_{1}$ and $U_{2}$ by a blue edge. We divide $W_{12}$ into $U_{1}^{*}, U_{2}^{*}$ so that the vertices of $U_{1}^{*}$ ( $U_{2}^{*}$ respectively) are adjacent to vertices of $U_{1}$ ( $U_{2}$ respectively) by green edges only. We do the same (except we use green instead of blue) to split $W_{34}$ into $U_{3}^{*}, U_{4}^{*}$ so that the vertices of $U_{3}^{*}$ ( $U_{4}^{*}$ respectively) are adjacent to all vertices of $U_{4}\left(U_{3}\right.$ respectively) by blue edges. This completes the proof of part (A). The proof of part (B) is exactly the same as in Case 1.

Lemma 11 also implies that any two vertices of $U_{1} \cup U_{2}$ or $U_{3} \cup U_{4}$ can be connected by a red path of length $n_{1}-1$. Hence, there is no red edge in $G\left[U_{1} \cup U_{2}\right]$ and $G\left[U_{3} \cup U_{4}\right]$.

The argument used to prove Claim 12 in Case 1 also yields that there are no two green independent edges between $\tilde{U}_{1}$ and $\tilde{U}_{2}$ and there are no two independent blue edges between $\tilde{U}_{3}$ and $\tilde{U}_{4}$. Hence, by removing the centers of one green and one blue star we obtain that $G\left[\tilde{U}_{1}, U_{2}\right]$ and $G\left[\tilde{U}_{2}, U_{1}\right]$ are blue and $t$-complete and $G\left[\tilde{U}_{3}, U_{4}\right]$ and $G\left[\tilde{U}_{4}, U_{3}\right]$ are green and $t$-complete. Moreover, there are no green edges between $U_{1}^{*}$ and $U_{2}^{*}$ and no blue edges between $U_{3}^{*}$ and $U_{4}^{*}$.

From Lemma 11 and from the $t$-completeness of $G\left[\tilde{U}_{1}, U_{2}\right]$ we conclude that any two vertices of $\tilde{U}_{1}$ are joined by a blue paths of length $n_{2}-5$ and $n_{2}-7$. Since $G\left[U_{3}^{*}, U_{3}\right]$ and $G\left[U_{3}\right]$ are $t$-complete and blue, we conclude that any two vertices of $\tilde{U}_{3}$ can be joined by a blue path of length 3 or 5 . Consequently, any two vertex disjoint blue edges between $\tilde{U}_{1}$ and $\tilde{U}_{3}$ would yield a blue cycle of length $n_{2}-$ a contradiction.

Hence, by symmetry, there are no two independent blue edges between $\tilde{U}_{i}$ and $\tilde{U}_{j}$ for $(i, j)=(1,3),(1,4),(2,3),(2,4)$. Similarly, there are no two independent green edges between $\tilde{U}_{i}$ and $\tilde{U}_{j}$ for $(i, j)=(1,3),(1,4),(2,3),(2,4)$. We remove at most 8 centers of green and blue stars and achieve that $G\left[\tilde{U}_{1} \cup \tilde{U}_{2}, \tilde{U}_{3} \cup \tilde{U}_{4}\right]$ is a red $t$-complete bipartite graph.

From Lemma 11 we immediately have that any two vertices in $\tilde{U}_{1} \cup \tilde{U}_{2}$ and any two vertices in $\tilde{U}_{3} \cup \tilde{U}_{4}$ can be joined by a red path of length $n_{1}-1$. Hence there are no red edges in $G\left[\tilde{U}_{1} \cup \tilde{U}_{2}\right]$ and in $G\left[\tilde{U}_{3} \cup \tilde{U}_{4}\right]$. Consequently, all the edges between $U_{1}^{*}$ and $U_{2}^{*}$ are blue and all the edges between $U_{3}^{*}$ and $U_{4}^{*}$ are green. It is easy to see now that the all edges in $U_{1}^{*}$ and $U_{2}^{*}$ are green and all edges in $U_{3}^{*}$ and $U_{4}^{*}$ are blue.

The second part of $(\mathrm{C})$ follows from the observation that in the above argument we ignored only edges incident to at most 10 vertices. 


\section{Structure of 3-colorings without long monochro- matic odd cycles}

In this section we prove Theorem 6 . First we state two lemmas.

\subsection{Some results about 2-colorings of graphs with large min- imum degree}

We know that $R\left(C_{n}, C_{n}\right)=2 n-1$ for every odd $n>4$. In [20], Łuczak needed a similar, but in some sense more general result on 2-colorings of "almost complete" graphs on slightly more than $2 n$ vertices.

Lemma 19 (Claim 8 in [20]). Let $0<\eta<10^{-5}$ and $n \geq n_{19}(\eta):=\exp \left(\eta^{-49}\right)$. Furthermore, let $G=G_{N}$ be a graph with $N=(2+\eta) n$ vertices and let

$$
e(G)>\left(\begin{array}{c}
N \\
2
\end{array}\right)-14 \eta^{3} n^{2}
$$

Then every 2-coloring of $G$ yields a monochromatic odd cycle of length at least $(1+$ $\eta / 10) n$.

We shall use an improved version of this lemma, in which it is strengthened in two ways: by adding structural stability and bringing down the size of $N$ from $(2+o(1)) n$ to $(3 / 2+o(1)) n$.

Lemma 20 (2-color stability lemma). There exists a positive integer $n_{20}$ with the following property. Let $n>n_{20}, s$ be any integer in $\left(20 n^{2 / 3}, n / 160\right)$, and $N=$ $3 n / 2+80 s$. Suppose that $G=G_{N}$ is (s/100)-complete. Then any 2-coloring of $G$ either contains a monochromatic odd cycle longer than $n+s$ or else $V(G)$ can be partitioned into $V_{1} \cup V_{2}$ so that

(a) all the edges of $G\left[V_{1}\right]$ and $G\left[V_{2}\right]$ are of the same color and $\left|V_{1}\right|,\left|V_{2}\right| \leq n+s$,

(b) all the edges of $G\left[V_{1}, V_{2}\right]$ are of the other color, with the possible exception of edges incident to one fixed vertex.

The proof of this lemma can be found in [15]. For complete graphs (i.e., when $s=0)$ this result was observed by Nikiforov and Schelp [21]. 


\section{2. Łuczak's even-odd decomposition}

If we know only that a graph $G$ does not contain odd cycles longer than $m$, then it easily can be the union of complete graphs of size at most $m$ and a bipartite graph. Euczak showed that up to some error the converse is also true.

Lemma 21 (Łuczak's decomposition lemma, Claim 7 in [20]). For every $0<\delta<$ $10^{-15}$ and $m, N$ satisfying

$$
\max \left\{2 \delta N, \frac{N}{\log (N) \delta^{16}}\right\}<m<N
$$

the following holds. If a graph $G=G_{N}$ contains no odd cycles longer than $m$, then $G$ contains two subgraphs $F$ and $B$ for which

(A) $V(F) \cup V(B)=V(G), V(B) \cap V(F)=\emptyset$, and each of $V(B)$ and $V(F)$ is either empty or contains at least $\delta m / 2$ vertices,

(B) $B$ is bipartite,

(C) $e(F) \leq \frac{1}{2} m|V(F)|$, and

(D) $e(G)-e(B)-e(F)<\delta N^{2}$.

Notice that Łuczak's decomposition lemma defines a natural decomposition of $V(G)$ into at most three sets: the vertex set of $F$, called the odd part, and the two sets of $B$, defining the even - or bipartite - part. We will refer to this decomposition as the Euczak decomposition.

\subsection{Proof of Theorem 6}

First we sketch the proof, then give the details.

Let $G$ be a graph on at least $(4-\eta) n$ vertices and with large minimum degree, let $\eta>0$ be sufficiently small, and let $n$ be sufficiently large. We consider a 3-coloring of $G$ with no monochromatic odd cycle longer than $(1+\eta / 100) n$. By symmetry, we may assume that the green subgraph has the smallest edge-set. Then we apply Lemma 21 to the red and blue subgraphs, obtaining the red Euczak decomposition $V_{1}$ (odd part), $V_{2}, V_{3}$ (bipartite part), and the blue Łuczak decomposition $W_{1}$ (odd part), $W_{2}, W_{3}$ (bipartite part). 


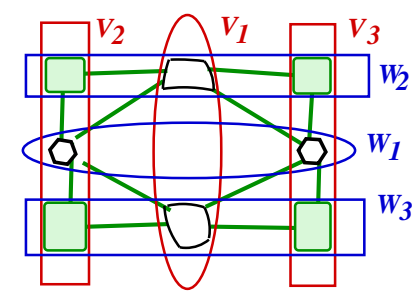

We shall distinguish three cases:

(a) The first case occurs when both sets $V_{2}, V_{3}$ in the red even part or both sets $W_{2}, W_{3}$ in the blue even part are "large" - larger than $(3 / 2+\eta) n$.

(b) The second case is when only one of $V_{2}, V_{3}$ or one of $W_{2}, W_{3}$, say $V_{2}$, is larger than $(3 / 2+\eta) n$ and $\left|V_{1}\right|+\left|V_{2}\right| \geq(2+\eta) n$.

(c) The last case covers the remaining part when $\left|V_{2}\right|,\left|V_{3}\right|,\left|W_{2}\right|,\left|W_{3}\right| \leq(3 / 2+$ $\eta) n$. This is the most involved case, requiring the most work.

In the first case, using the 2-color stability lemma, Lemma 20, we show that the subgraph of $G$ induced by the red even part or by the blue even part is basically an $\mathrm{EC}_{1}(\lceil(1 / 2+\eta / 4) n\rceil)$ or $\mathrm{EC}_{2}((\lceil 1 / 2+\eta / 4) n\rceil)$.

In the second case, again by using Lemma 20, we show that the subgraph of $G$ induced by $V_{1} \cup V_{2}$ contains a monochromatic (blue or green) odd cycle longer that $(1+\eta / 100) n$, which is a contradiction with our assumptions on the original 3 -coloring.

In the last case we find a monochromatic odd cycle longer that $(1+\eta / 100) n$ by patching together long paths in monochromatic dense subgraphs of $G$ induced by the sets $V_{i} \cap W_{j}$, which is again a contradiction with our assumptions on the original 3 -coloring.

Let us turn to the details. We set $\eta_{6}:=10^{-5}$ and, for a given $0<\eta \leq \eta_{6}$, we let $n_{6}:=\exp \left(8 \eta^{-128}\right)$. Suppose that $n>n_{6},(4-\eta) n \leq N<(4+\eta) n$ and $G=G_{N}$ is a graph with minimum degree $\delta(G) \geq\left(1-\eta^{4}\right) N$. Let $G=G^{r} \cup G^{b} \cup G^{g}$ be an arbitrary 3-coloring of its edges by red, blue and green, with

$$
\text { no monochromatic odd cycle longer than }\left(1+\frac{\eta}{100}\right) n \text {. }
$$

Without loss of generality we may assume that $e\left(G^{r}\right) \geq e\left(G^{b}\right) \geq e\left(G^{g}\right)$. Hence

$$
e\left(G^{r b}\right)=e\left(G^{r}\right)+e\left(G^{b}\right) \geq \frac{2 e(G)}{3} \geq\left(1-\eta^{4}\right) \frac{N(N-1)}{3} .
$$

We apply the Euczak decomposition lemma, Lemma 21, with $\delta=\eta^{8}$ and $m=$ $(1+\eta / 100) n$ to the red graph $G^{r}$ and obtain a decomposition of $V(G)$ into $V_{1}, V_{2}$ and 
$V_{3}$ and two subgraphs $F^{r}$ and $B^{r}$ so that the vertex set of $F^{r}$ is $V_{1}, B^{r}$ is bipartite with bipartition $V_{2} \cup V_{3}$,

$$
e\left(F^{r}\right) \leq \frac{1}{2}\left(1+\frac{\eta}{100}\right) n\left|V_{1}\right|
$$

and

$$
e\left(G^{r}\right)-e\left(F^{r}\right)-e\left(B^{r}\right)<\eta^{8} N^{2} .
$$

Similarly, we apply Lemma 21 to the blue graph $G^{b}$ and obtain the decomposition of $V(G)$ into $W_{1}, W_{2}$ and $W_{3}$ and two subgraphs $F^{b}$ and $B^{b}$ so that the vertex set of $F^{b}$ is $W_{1}, B^{b}$ is bipartite with bipartition $W_{2} \cup W_{3}$,

$$
e\left(F^{b}\right) \leq \frac{1}{2}\left(1+\frac{\eta}{100}\right) n\left|W_{1}\right|
$$

and

$$
e\left(G^{b}\right)-e\left(F^{b}\right)-e\left(B^{b}\right)<\eta^{8} N^{2} .
$$

Note that after removing at most $\eta^{8} N^{2}$ red edges and $\eta^{8} N^{2}$ blue edges from the original 3-coloring

(A) the graphs induced by $V_{2}$ and by $V_{3}$ and the bipartite graph induced by $V_{1}$ and $V_{2} \cup V_{3}$ are 2-colored by blue and green only,

(B) the graphs induced by $W_{2}$ and by $W_{3}$ and the bipartite graph induced by $W_{1}$ and $W_{2} \cup W_{3}$ are 2-colored by red and green only,

(C) for $i, j \in\{2,3\}$, the graphs induced by $V_{i} \cap W_{j}$ and the bipartite graphs induced by $V_{1} \cap W_{1}$ and $V_{i} \cap W_{j}$, are completely green.

Furthermore, there are at most $2 \eta^{4} N$ vertices incident to more than $2 \eta^{4} N$ edges removed above. We delete these vertices and obtain

(D) a $\left\lceil 3 \eta^{4} N\right\rceil$-complete subgraph of $G$ with at least $(4-2 \eta) n$ vertices. Without loss of generality we identify $G$ with this subgraph, at least in this part of the proof.

We distinguish three cases, as discussed in the outline of this proof. 


\subsection{Either both $V_{2}$ and $V_{3}$ or both $W_{2}$ and $W_{3}$ are "large"}

Throughout this section we will assume that

$$
\left|V_{2}\right|,\left|V_{3}\right|>\left(\frac{3}{2}+\eta\right) n
$$

(The case when $\left|W_{2}\right|,\left|W_{3}\right|>(3 / 2+\eta) n$ is handled in the same way.) By (A) and (D) we know that for the blue-green graph $G^{b g}$,

$$
\delta\left(G^{b g}\left[V_{i}\right]\right) \geq\left|V_{i}\right|-3 \eta^{4} N \quad \text { for } \quad i=2,3 .
$$

We apply Lemma 20 with $s=\eta / 100$ to $G^{b g}\left[V_{i}\right]$ for $i=2,3$. Since $G^{b g}\left[V_{i}\right]$ does not contain any monochromatic odd cycles longer than $(1+\eta / 100) n$, there must be a partition $V_{i 1} \cup V_{i 2}$ of $V_{i}$ such that $G^{b g}\left[V_{i 1}\right]$ and $G^{b g}\left[V_{i 2}\right]$ are monochromatic in the same color (blue or green), and $G^{b g}\left[V_{i 1}, V_{i 2}\right]$ is monochromatic in the other color, with a possible exception of some edges incident to one vertex. We remove this vertex.

By (D), for the monochromatic graph $G\left[V_{i j}\right]=G^{b g}\left[V_{i j}\right]$,

$$
\delta\left(G^{b g}\left[V_{i j}\right]\right) \geq\left|V_{i j}\right|-3 \eta^{4} N \quad \text { for } i=2,3 \text {, and } j=1,2 .
$$

Suppose $\left|V_{i j}\right|>(1+\eta / 2) n$. Since $\delta\left(G\left[V_{i j}\right]\right) \geq\left|V_{i j}\right|-3 \eta^{4} N>\left|V_{i j}\right| / 2$, by Lemma 9 , $G\left[V_{i j}\right] \subset G$ contains a monochromatic odd cycle longer than $\left|V_{i j}\right|-2 \geq(1+\eta / 100) n$, which is a contradiction.

Hence each of four vertex sets $V_{21}, V_{22}, V_{31}, V_{32}$ must be smaller than $(1+\eta / 2) n$ which in turn implies that each of these sets must have size at least $(1 / 2+\eta / 2) n$. We need two consider two sub-cases:

(a) the induced subgraphs $G^{b g}\left[V_{21}\right], G^{b g}\left[V_{22}\right]$, and $G^{b g}\left[V_{31}, V_{32}\right]$ are green and $G^{b g}\left[V_{31}\right], G\left[V_{32}\right]$ and $G^{b g}\left[V_{21}, V_{22}\right]$ are blue;

(b) the induced subgraphs $G^{b g}\left[V_{21}\right], G^{b g}\left[V_{22}\right], G^{b g}\left[V_{31}\right], G^{b g}\left[V_{32}\right]$ are green, and $G^{b g}\left[V_{21}, V_{22}\right]$ and $G^{b g}\left[V_{31}, V_{32}\right]$ are blue.

Observe that in both sub-cases, by Lemmas 10 and 11 applied with $t=\left\lceil 3 \eta^{4} N\right\rceil$,

(E) any two vertices in $V_{i j}$ are connected (in $G^{b g}\left[V_{i j}\right]$ ) by a monochromatic path of length $\ell$ for every $2 \leq \ell \leq(1+\eta / 4) n$;

(F) every vertex in $V_{i 1}$ is connected (in $G^{b g}\left[V_{i 1}, V_{i 2}\right]$ ) to every vertex in $V_{i 2}$ by a monochromatic path of length $\ell$ for every odd $3 \leq \ell \leq(1+\eta / 4) n$ and $i=2,3$; 
(G) any two vertices in $V_{i 1}$ are connected (in $G^{b g}\left[V_{i 1}, V_{i 2}\right]$ ) by a monochromatic path of length $\ell$ for every even $2 \leq \ell \leq(1+\eta / 4) n$ and $i=2,3$.

Statements $(\mathrm{E})-(\mathrm{G})$ remain true after removing arbitrary set of $\eta n / 10$ vertices from $G$.

\subsubsection{Sub-case (a): Distinct color patterns}
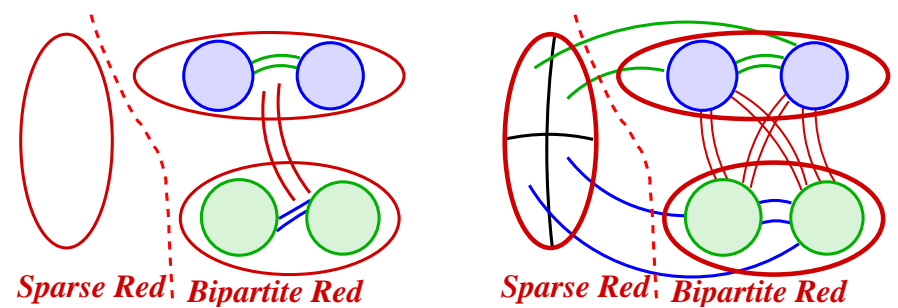

In this sub-case, there are no two independent blue or green edges in $G\left[V_{2 i}, V_{3 j}\right]$ for any $i, j \in\{1,2\}$. Indeed, if there were two blue edges $u v$ and $u^{\prime} v^{\prime}$ with $u \neq u^{\prime} \in V_{2 i}$ and $v \neq v^{\prime} \in V_{3 j}$, then using $(\mathrm{G})$ and (E) we could find a blue even $\left(u, u^{\prime}\right)$-path of length at least $(1+\eta / 100) n$ in $G\left[V_{21}, V_{22}\right]$ and also a blue $\left(v, v^{\prime}\right)$-path of length 3 in $G\left[V_{3 j}\right]$. These two paths, $u v$ and $u^{\prime} v^{\prime}$, would form an odd blue cycle longer than $(1+\eta / 100) n-$ a contradiction. We would obtain a contradiction for two independent green edges similarly.

Hence each of the four graphs $G\left[V_{2 i}, V_{3 j}\right]$ contains at most one green star, one blue star, and all the other edges are red. We remove the 8 centers of these stars together with all the edges incident to them. All the edges of the remaining graph $G\left[V_{21} \cup V_{22}, V_{31} \cup V_{32}\right]$ are red and, by (D), this graph is still $\left\lceil 3 \eta^{4} N\right\rceil$-complete. Since $\left|V_{21} \cup V_{22}\right|,\left|V_{31} \cup V_{32}\right|>3 n / 2$, Lemma 11, applied with $t=\left\lceil 3 \eta^{4} N\right\rceil$, implies that any two vertices of $V_{21} \cup V_{22}$ and $V_{31} \cup V_{32}$ are connected by an even red path of length $(1+\eta) n$. Hence there are no red edges in $G\left[V_{21} \cup V_{22}\right]$ neither in $G\left[V_{31} \cup V_{32}\right]$.

Clearly, by removing vertices, we can reduce each $V_{i j}$ to size $\lceil(1 / 2+\eta / 4) n\rceil$ and these four sets induce a coloring embeddable into $\mathrm{EC}_{2}(\lceil(1 / 2+\eta / 4) n\rceil)$. This was to be proven. 


\subsubsection{Sub-case (b): The same color patterns}

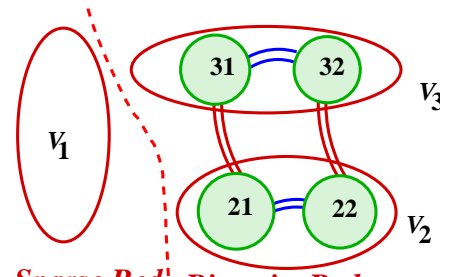

Sparse Red: Bipartite Red

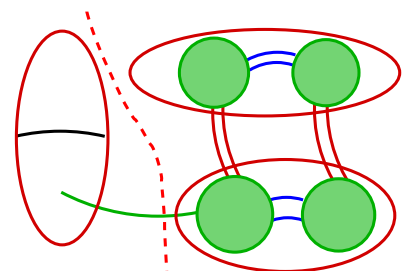

Sparse Red: Bipartite Red

Similarly to Sub-case (a), there are no two independent green edges between $V_{2 i}$ and $V_{3 j}, i, j \in\{1,2\}$. Hence, by removing the centers of at most 4 green stars, we achieve that all the edges of $G\left[V_{21} \cup V_{22}, V_{31} \cup V_{32}\right]$ are red and blue only.

By $(\mathrm{F})$, there is an odd blue path of length at least $(1+\eta / 100) n$ between any vertex in $V_{21}$ and any vertex in $V_{22}\left(V_{31}\right.$ and $V_{32}$, respectively). Since there is no blue odd cycle longer than $(1+\eta / 100) n$, no vertex of $V_{3}\left(V_{2}\right.$, respectively) is adjacent to both $V_{21}$ and $V_{22}$ ( $V_{31}$ and $V_{32}$, respectively) by a blue edge. Furthermore, suppose there are vertices $u, v \in V_{21}, u^{\prime} \in V_{31}, v^{\prime} \in V_{32}$ such that $u u^{\prime}$ and $v v^{\prime}$ are both blue. By $(\mathrm{G})$, there exists a $w \in V_{22}$ such that $u w$ and $v w$ are blue and, by $(\mathrm{F})$, there is an odd blue $\left(u^{\prime}, v^{\prime}\right)$-path of length at least $(1+\eta / 100) n$ in $G\left[V_{31}, V_{32}\right]$. This path and $u u^{\prime}, v v^{\prime}, u w$ and $v w$ form a blue odd cycle longer than $(1+\eta / 100) n$, which is a contradiction.

Thus, by symmetry, the graphs $G\left[V_{2 i}, V_{3 j}\right], i, j \in\{1,2\}$, are monochromatic and either $G\left[V_{21}, V_{31}\right]$ and $G\left[V_{22}, V_{32}\right]$ or $G\left[V_{21}, V_{32}\right]$ and $G\left[V_{22}, V_{31}\right]$ have red edges only. As before, we can reduce the size of each $V_{i j}$ to $\lceil(1 / 2+\eta / 4) n\rceil$ and these four sets induce a coloring embeddable into $\mathrm{EC}_{1}(\lceil(1 / 2+\eta / 4) n\rceil)$.

\subsection{Exactly one of $V_{2}, V_{3}$ or one of $W_{2}, W_{3}$ is "large"}

Throughout this section we will assume that

$$
\left|V_{2}\right|>(3 / 2+\eta) n,\left|V_{3}\right| \leq(3 / 2+\eta) n \quad \text { and } \quad\left|V_{1}\right|+\left|V_{2}\right|>(2+\eta) n .
$$

We start exactly as in Section 3.4 and apply Lemma 20 (with $s=\lceil\eta n / 100\rceil$ ) to $G^{b g}\left[V_{2}\right]$. Since $G^{b g}\left[V_{2}\right]$ does not contain any monochromatic odd cycles longer than $(1+\eta / 100) n$, there must be a partition $V_{21} \cup V_{22}$ of $V_{2}$ such that $G^{b g}\left[V_{21}\right]$ and $G^{b g} G\left[V_{22}\right]$ are monochromatic in the same color (say, green), and $G^{b g}\left[V_{21}, V_{22}\right]$ is monochromatic in blue, with a possible exception of some edges incident to one 
vertex that we remove. Moreover, $V_{21}, V_{22}$ satisfy

$$
\left(\frac{1}{2}+\frac{\eta}{2}\right) n \leq\left|V_{21}\right|,\left|V_{22}\right| \leq\left(1+\frac{\eta}{2}\right) n
$$

and also, by Lemmas 10 and 11 applied with $t=\left\lceil 3 \eta^{4} N\right\rceil$ (cf. Section 3.4),

(E') any two vertices in $V_{2 j}$ are connected (in $G^{b g}\left[V_{2 j}\right]$ ) by a green path of length $\ell$ for every $2 \leq \ell \leq(1+\eta / 4) n$ and $j=2,3$;

(F') every vertex in $V_{21}$ is connected (in $G^{b g}\left[V_{21}, V_{22}\right]$ ) to every vertex in $V_{22}$ by a blue path of length $\ell$ for every odd $3 \leq \ell \leq(1+\eta / 4) n$;

$\left(\mathrm{G}^{\prime}\right)$ any two vertices in $V_{2 j}$ are connected (in $G^{b g}\left[V_{21}, V_{22}\right]$ ) by a blue path of length $\ell$ for every even $2 \leq \ell \leq(1+\eta / 4) n$ and $j=2,3$.

Statements $\left(E^{\prime}\right)-\left(G^{\prime}\right)$ again remain true after removing arbitrary set of $\eta n / 10$ vertices from $G$.

By (D), $G^{b g}\left[V_{1}, V_{2}\right]$ is a $\left\lceil 3 \eta^{4} N\right\rceil$-complete bipartite graph. Suppose that one of the vertices $v \in V_{1}$ has blue neighbors $u \in V_{21}$ and $u^{\prime} \in V_{22}$. By (F'), $u$ and $u^{\prime}$ are connected in $G^{b g}\left[V_{21}, V_{22}\right]$ by a blue odd path of length at least $(1+\eta / 100) n$. This path and $v u, v u^{\prime}$ form an odd blue cycle longer than $(1+\eta / 100) n$ - a contradiction. Therefore, each $v \in V_{1}$ has either only green neighbors in $V_{21}$ or only green neighbors in $V_{22}$. We split $V_{1}$ into two sets $V_{11}$ and $V_{12}$ such that there are only green edges between $V_{1 j}$ and $V_{2 j}$. Since

$$
\left|V_{11}\right|+\left|V_{12}\right|+\left|V_{21}\right|+\left|V_{22}\right|=\left|V_{1}\right|+\left|V_{2}\right| \geq(2+\eta) n
$$

either $\left|V_{11}\right|+\left|V_{21}\right| \geq(1+\eta / 2) n$ or $\left|V_{12}\right|+\left|V_{22}\right| \geq(1+\eta / 2) n$. Without loss of generality, we may assume that $\left|V_{12}\right|+\left|V_{22}\right| \geq(1+\eta / 2) n$.

Now we find a green odd cycle of length longer than $(1+\eta / 100) n$ in $V_{12} \cup V_{22}$. Since $G^{g}\left[V_{12}, V_{22}\right]$ is $\left\lceil 3 \eta^{4} N\right\rceil$-complete bipartite graph, by Lemma 11 there exists a green even $\left(v, v^{\prime}\right)$-path $P$ of length at least $2 \min \left\{\left|V_{22}\right|-\eta n / 8,\left|V_{12}\right|-2\left\lceil 3 \eta^{4} N\right\rceil\right\}$ for some $v, v^{\prime} \in V_{22}$. Set $P^{-}=P \backslash\left\{v, v^{\prime}\right\}$ and notice that, by (D), $G^{g}\left[V_{22} \backslash V\left(P^{-}\right)\right]$is $\left\lceil 3 \eta^{4} N\right\rceil$-complete. Furthermore,

$$
\frac{\left|V_{22} \backslash V\left(P^{-}\right)\right|}{2}-2 \geq \frac{\left|V_{22}\right|-\left(\left|V_{22}\right|-\frac{\eta n}{8}\right)}{2}-2>\left\lceil 3 \eta^{4} N\right\rceil .
$$

Therefore, by Lemma 10, the graph $G^{g}\left[V_{22} \backslash V\left(P^{-}\right)\right]$contains a green odd $\left(v, v^{\prime}\right)$-path $P^{\prime}$ of length at least $\left|V_{22} \backslash V(P)\right|-1$. Thus, $P \cup P^{\prime}$ is an odd green cycle of length 
at least

$$
\begin{aligned}
& 2 \min \left\{\left|V_{22}\right|-\frac{\eta n}{8},\left|V_{12}\right|-2\left\lceil 3 \eta^{4} N\right\rceil\right\}+\left|V_{22} \backslash V(P)\right|-1 \\
= & \left|V_{22}\right|+\min \left\{\left|V_{22}\right|-\frac{\eta n}{8}-1,\left|V_{12}\right|-2\left\lceil 3 \eta^{4} N\right\rceil-1\right\} \\
\geq & \min \left\{\left|V_{22}\right|+\left|V_{12}\right|, 2\left|V_{22}\right|\right\}-\frac{\eta n}{7} .
\end{aligned}
$$

By our assumptions we have $\left|V_{22}\right|+\left|V_{12}\right|-\eta n / 7 \geq(1+\eta / 4) n-\eta n / 7>(1+\eta / 100) n$ and $2\left|V_{22}\right|-\eta n / 7 \geq 2(1 / 2+\eta / 2) n-\eta n / 7>(1+\eta / 100) n$. Hence, $P \cup P^{\prime}$ is an odd green cycle of length at least $(1+\eta / 100) n$, which is again a contradiction.

The case when $\left|W_{i}\right|>(3 / 2+\eta) n$ and $\left|W_{5-i}\right| \leq(3 / 2+\eta) n, i=2,3$, is handled similarly.

\section{6. $V_{2}, V_{3}, W_{2}, W_{3}$ are all "small"}

Now we deal with the last case. It is impossible to have $\left|V_{2}\right|>(3 / 2+\eta) n,\left|V_{3}\right| \leq$ $(3 / 2+\eta) n$, and $\left|V_{1}\right|+\left|V_{2}\right| \leq(2+\eta) n$ because, by (D), $\left|V_{1}\right|+\left|V_{2}\right|+\left|V_{3}\right| \geq(4-2 \eta) n$. Hence,

$$
\left|V_{2}\right|,\left|V_{3}\right| \leq\left(\frac{3}{2}+\eta\right) n
$$

must hold, and, consequently,

$$
\left|V_{1}\right| \geq(4-2 \eta) n-2\left(\frac{3}{2}+\eta\right) n=(1-4 \eta) n
$$

By the same argument, we also have $\left|W_{1}\right| \geq(1-4 \eta) n$ and $\left|W_{2}\right|,\left|W_{3}\right| \leq(3 / 2+\eta) n$.

Let $S:=\left(V_{2} \cap W_{2}\right) \cup\left(V_{2} \cap W_{3}\right) \cup\left(V_{3} \cap W_{2}\right) \cup\left(V_{3} \cap W_{3}\right)$ and $Q:=V_{1} \cap W_{1}$. We also set $\sigma:=|S| / n$ and $\tau:=|Q| / n$ and make the following observation.

\section{Claim 22.}

(i) $\sigma \geq 0.48$;

(ii) if $\tau \geq \sigma$, then $\sigma \geq 0.66$;

(iii) if $\tau, \sigma \geq 1 / 2+\eta$, then there is an odd green cycle of length $(1+\eta / 100) n$.

The proof of Claim 22 is deferred to Section 3.6.3. Now we show how Claim 22 implies the existence of an odd monochromatic cycle longer than $(1+\eta / 100) n$. We distinguish two sub-cases. 


\subsubsection{Sub-case (a): $\sigma>1+35 \eta$}

We call $V_{i} \cap W_{j}, i, j \in\{2,3\}$, "negligible" if $\left|V_{i} \cap W_{j}\right|<11 \eta n$ and "non-negligible" otherwise. By (C) and (D), $G\left[V_{i} \cap W_{j}\right]$ is a green $t$-complete graph with $t=\left\lceil 3 \eta^{4} N\right\rceil$. Since each "non-negligible" set $V_{i} \cap W_{j}$ has at least $11 \eta n>2 t+2$ vertices, Theorem 9 applies. In particular, each "non-negligible" set $V_{i} \cap W_{j}$ contains an odd green cycle of length at least $\left|V_{i} \cap W_{j}\right|-1$. Since $G\left[V_{i} \cap W_{j}\right]$ contains no odd green cycle longer than $(1+\eta / 100) n$, we must have $\left|V_{i} \cap W_{j}\right|<(1+\eta / 50) n$. As $\sigma>1+35 \eta$, there are at least 2 "non-negligible" sets $V_{i} \cap W_{j}$.

(a1) Suppose first that $\tau>\eta$ and all four sets $V_{i} \cap W_{j}$ are "non-negligible". As each $G^{g}\left[Q, V_{i} \cap W_{j}\right]$ is a $t$-complete graph (see (C) and (D)) and $|Q|,\left|V_{i} \cap W_{j}\right|>$ $t+2$, where $t=\left\lceil 3 \eta^{4} N\right\rceil$, there are six distinct vertices $q_{22}, q_{23}, q_{32}, q_{33} \in Q$ and $u_{i j}, v_{i j} \in V_{i} \cap W_{j}, i, j \in\{2,3\}$ for which all the edges $q_{22} u_{22}, v_{22} q_{23}, q_{23} u_{23}, v_{23} q_{33}$, $q_{33} u_{33}, v_{33} q_{32}, q_{32} u_{32}, v_{32} q_{22}$ are green. Since $\left|V_{i} \cap W_{j}\right| \geq 11 \eta n>2 t+4$, Lemma 10 applies and each $G^{g}\left[V_{i} \cap W_{j}\right]$ contains a path $P\left(u_{i j}, v_{i j}\right)$ of length at least $\left|V_{i} \cap W_{j}\right|-1$ and such that $\left|P\left(u_{22}, v_{22}\right)\right|+\left|P\left(u_{23}, v_{23}\right)\right|+\left|P\left(u_{32}, v_{32}\right)\right|+\left|P\left(u_{33}, v_{33}\right)\right|$ is odd. These four paths and eight edges form an odd green cycle of length at least $\sigma n-3 \cdot 11 \eta n-4>$ $(1+\eta / 100) n$. The same argument works when we have two or three "non-negligible" sets only.

(a2) Assume now that $\tau \leq \eta$. From (12) we conclude that

$$
\left|V_{1} \backslash Q\right|,\left|W_{1} \backslash Q\right| \geq(1-5 \eta) n .
$$

Notice that, by (A) and (B),

$$
G\left[V_{1} \backslash Q, W_{1} \backslash Q\right] \subset G\left[V_{1}, V_{2} \cup V_{3}\right] \cap G\left[W_{2} \cup W_{3}, W_{1}\right]
$$

is a green bipartite graph. By (D), it is also $t$-complete. Therefore Lemma 11 implies that any two vertices in $V_{1} \backslash Q$ or in $W_{1} \backslash Q$ are joined by an even green path of length at least $(2-10 \eta) n-4 t>3 n / 2$.

This means that if we find two green independent edges between any "nonnegligible" set $V_{i} \cap W_{j}$ and $V_{1} \backslash Q$ or $W_{1} \backslash Q$, then we join their endpoints in $V_{i} \cap V_{j}$ by a path of length 3 and the endpoints in $V_{1} \backslash Q$ (or in $W_{1} \backslash Q$ ) by an even green path of length at least $3 n / 2$, thus obtaining an odd green cycle of length at least $3 n / 2>(1+\eta / 100) n$.

By symmetry, we may assume that $V_{2} \cap W_{2}$ is a "non-negligible" set, i.e., $\mid V_{2} \cap$ $W_{2} \mid \geq 11 \eta n \geq 2\left\lceil 3 \eta^{4} N\right\rceil$. If either $\left|V_{2} \cap W_{1}\right|$ or $\left|W_{2} \cap V_{1}\right|$ is at least $11 \eta n$, then there 
are two green independent edges in either $G\left[V_{2} \cap W_{2}, V_{1} \cap W_{2}\right]$ or $G\left[V_{2} \cap W_{2}, V_{2} \cap W_{1}\right]$ because both graphs are green (by (A) and (B)) and $\left\lceil 3 \eta^{4} N\right\rceil$-complete (see (D)).

Otherwise, we have $\left|V_{2} \cap W_{1}\right|,\left|W_{2} \cap V_{1}\right| \leq 11 \eta n$. Since $\left|V_{1} \backslash Q\right|,\left|W_{1} \backslash Q\right| \geq(1-5 \eta) n$, we get

$$
\left|V_{3} \cap W_{1}\right|=\left|W_{1} \backslash Q\right|-\left|V_{2} \cap W_{1}\right| \geq 11 \eta n \geq 2\left\lceil 3 \eta^{4} N\right\rceil
$$

and

$$
\left|W_{3} \cap V_{1}\right|=\left|V_{1} \backslash Q\right|-\left|W_{2} \cap V_{1}\right| \geq 11 \eta n \geq 2\left\lceil 3 \eta^{4} N\right\rceil .
$$

There exist at least two "non-negligible" sets, thus one of the sets $V_{2} \cap W_{3}, V_{3} \cap W_{2}$, $V_{3} \cap W_{3}$ must be "non-negligible". The bipartite graphs $G\left[V_{2} \cap W_{3}, V_{1} \cap W_{3}\right], G\left[V_{3} \cap\right.$ $\left.W_{2}, V_{3} \cap W_{1}\right], G\left[V_{3} \cap W_{3}, V_{3} \cap W_{1}\right]$ are all green (by (A) and (B)) and $\left\lceil 3 \eta^{4} N\right\rceil$-complete (by $(\mathrm{D}))$. Hence any one of them whose both partition sets are "non-negligible" contains two green independent edges.

\subsubsection{Sub-case (b): $0.48<\sigma \leq 1+35 \eta$}

Claim 22(iii) implies that we cannot have $\sigma, \tau \geq 1 / 2+\eta$. By (ii) of Claim 22, we cannot have $\sigma \leq 1 / 2+\eta \leq \tau$ either. Hence we may assume that $\tau<1 / 2+\eta$, and, therefore, $|Q|=\tau n<(1 / 2+\eta) n$. (12) implies that $\left|V_{1} \backslash Q\right|,\left|W_{1} \backslash Q\right| \geq(1 / 2-5 \eta) n$.

If there are at least two "non-negligible" sets $V_{i} \cap W_{j}, i, j \in\{2,3\}$, then we obtain an odd green cycle of length at least $(1+\eta / 100) n$ similarly to (a2) above: first we find two green vertex disjoint edges $e, f$ between some "non-negligible" set $V_{i} \cap W_{j}$ and $V_{1} \backslash Q$ or $W_{1} \backslash Q$. Then we join their endpoints in $V_{i} \cap V_{j}$ by a green odd path $P$ of length at least $\left|V_{i} \cap W_{j}\right|-1$ in a green $\left\lceil 3 \eta^{4} N\right\rceil$-complete graph $G\left[V_{i} \cap W_{j}\right]$ (see (C) and (D)). We also connect the endpoints of $e, f$ in $V_{1} \backslash Q$ (or in $W_{1} \backslash Q$ ) by an even green path $P^{\prime}$ of length at least $2(1 / 2-5 \eta) n-4\left\lceil 3 \eta^{4} N\right\rceil$. Clearly, $P \cup P^{\prime} \cup\{e, f\}$ is an odd green cycle of length at least

$\left|V_{i} \cap W_{j}\right|-1+2\left(\frac{1}{2}-5 \eta\right) n-4\left\lceil 3 \eta^{4} N\right\rceil>11 \eta n+(1-10 \eta) n-5\left\lceil 3 \eta^{4} N\right\rceil>\left(1+\frac{\eta}{100}\right) n$.

Thus, by symmetry, we may assume that $\left|V_{2} \cap W_{2}\right|>\sigma n-3 \cdot 11 \eta n$ is the only "non-negligible" set. If $\left|V_{1} \cap W_{2}\right| \geq \eta n / 2$ or $\left|W_{1} \cap V_{2}\right| \geq \eta n / 2$, then we again proceed as above to obtain a green odd cycle longer than $(1+\eta / 100) n$.

Consequently, we also assume $\left|V_{1} \cap W_{2}\right|,\left|W_{1} \cap V_{2}\right| \leq \eta n / 2$. Since $|V|=|S|+$ $|Q|+\left|V_{1} \cap W_{2}\right|+\left|W_{1} \cap V_{2}\right|+\left|V_{1} \cap W_{3}\right|+\left|W_{1} \cap V_{3}\right|$, we get

$$
\left|V_{1} \cap W_{3}\right|+\left|W_{1} \cap V_{3}\right| \geq(4-2 \eta) n-\sigma n-\tau n-\eta n-2 \frac{\eta n}{2}>(2.5-40 \eta) n .
$$


Since $\left|V_{3}\right|,\left|W_{3}\right| \leq(3 / 2+\eta) n$ by $(11)$, we obtain $(1-41 \eta) n,\left|V_{1} \cap W_{3}\right| \geq(1-41 \eta) n$. The induced bipartite subgraph $G\left[V_{1} \cap W_{3}, W_{1} \cap V_{3}\right] \subset G\left[V_{1}, V_{2} \cup V_{3}\right] \cap G\left[W_{1}, W_{2} \cup W_{3}\right]$ has all its edges green by $(\mathrm{A})$ and $(\mathrm{B})$, and it is also $\left\lceil 3 \eta^{4} N\right\rceil$-complete by (D). Lemma 11 implies that any two vertices of $W_{1} \cap V_{3}$ ( $V_{1} \cap W_{3}$, respectively) are connected by an even path of length at least $2((1-41 \eta) n)-4\left\lceil 3 \eta^{4} N\right\rceil>(1+\eta / 100) n$. Hence, there are no green edges in $W_{1} \cap V_{3}$ and in $V_{1} \cap W_{3}$.

By (A), there are no red edges in $W_{1} \cap V_{3}$ either. Hence, by (D), $G\left[W_{1} \cap V_{3}\right]$ is a $\left\lceil 3 \eta^{4} N\right\rceil$-complete blue graph. Since $2\left\lceil 3 \eta^{4} N\right\rceil<(1-41 \eta) n \leq(1-41 \eta) n$, Theorem 9 applies and $G\left[W_{1} \cap V_{3}\right]$ contains green cycles of all lengths between 3 and $\left|W_{1} \cap V_{3}\right|$. Using (5) we obtain that $\left|W_{1} \cap V_{3}\right| \leq(1+\eta / 100) n+2$.

Similarly, using the red $\left\lceil 3 \eta^{4} N\right\rceil$-complete graph $G\left[W_{3} \cap V_{1}\right]$, we obtain $\left|W_{3} \cap V_{1}\right| \leq$ $(1+\eta / 100) n+2$. Equation $|V|=|S|+|Q|+\left|V_{1} \cap W_{2}\right|+\left|W_{1} \cap V_{2}\right|+\left|V_{1} \cap W_{3}\right|+\left|W_{1} \cap V_{3}\right|$ yields

$$
\sigma n \geq(4-2 \eta) n-\tau n-2 \frac{\eta n}{2}-2\left(1+\frac{\eta}{100}\right) n-4 \geq\left(\frac{3}{2}-5 \eta\right) n
$$

We know that $\left|V_{2} \cap W_{2}\right| \geq \sigma n-33 \eta n \geq(3 / 2-38 \eta) n$. Since $G\left[V_{2} \cap W_{2}\right]$ is a green $\left\lceil 3 \eta^{4} N\right\rceil$-complete graph (see $(\mathrm{C})$ and $(\mathrm{D})$ ), by Theorem 9 , it contains a green odd cycle of length at least $\left|V_{2} \cap W_{2}\right|-1 \geq(3 / 2-38 \eta) n-1>(1+\eta / 100) n$. This is again a contradiction with (5).

What remains to prove is Claim 22.

\subsubsection{Proof of Claim 22}

To get (i), first we need an upper bound on $e\left(G^{r b}\right)$. By $(\mathrm{A})$, there are no red edges between $V_{1}$ and $W_{1} \backslash V_{1}$ or in $W_{1} \backslash V_{1}$. Similarly, by (B), there are no blue edges between $W_{1}$ and $V_{1} \backslash W_{1}$ or in $V_{1} \backslash W_{1}$. Thus, by (7) and (9), there are at most

$$
\begin{aligned}
\frac{1}{2}\left(1+\frac{\eta}{100}\right) n\left|V_{1}\right|+\frac{1}{2}\left(1+\frac{\eta}{100}\right) n\left|W_{1}\right| & \leq 2 \cdot\left(1+\frac{\eta}{100}\right) n \frac{\left|V_{1} \cup W_{1}\right|}{2} \\
& \leq\left(1+\frac{\eta}{100}\right) n(N-\sigma n)
\end{aligned}
$$

red and blue edges in $V_{1} \cup W_{1}$. We have at most

$$
\sum_{i, j \in\{2,3\}}\left(\left|V_{2} \cap W_{i}\right|\left|V_{3} \cap W_{j}\right|+\left|W_{2} \cap V_{i}\right|\left|W_{3} \cap V_{j}\right|\right) \leq \frac{\sigma^{2} n^{2}}{2}
$$


red and blue edges in $V(G) \backslash\left(V_{1} \cup W_{1}\right)$ and the number of (potentially) red and blue edges between $S$ and $V_{1} \cup W_{1} \backslash Q$ is bounded by

$$
\sigma n(N-\sigma n-\tau n) \text {. }
$$

Finally, by (8) and (10), the number of red and blue edges not included above is bounded by $2 \eta^{8} N^{2}$. From this we obtain that

$$
e\left(G^{r b}\right) \leq\left(1+\frac{\eta}{100}\right) n(N-\sigma n)+\frac{\sigma^{2} n^{2}}{2}+\sigma n(N-\sigma n-\tau n)+2 \eta^{8} N^{2} .
$$

This upper bound is decreasing in $\tau$, hence using $\tau=0$ and combining it with (6) and $(4-\eta) \leq N \leq(4+\eta) n$ results in a quadratic inequality that yields $\sigma>0.48$ for every $\eta<10^{-3}$. To obtain (ii), we just replace $\tau$ by $\sigma$ (instead of 0 ) and proceed as above.

Now we prove (iii). It follows from (C) and (D) that $G^{g}\left[Q, V_{i} \cap W_{j}\right]$ and $G^{g}\left[V_{i} \cap\right.$ $\left.W_{j}\right]$ are $\left\lceil 3 \eta^{4} N\right\rceil$-complete graphs; therefore, $G^{g}[Q, S]$ is also $\left\lceil 3 \eta^{4} N\right\rceil$-complete. We assume that $\sigma \geq(1 / 2+\eta) n$, therefore at least one set $V_{i} \cap W_{j}$ must have size at least $n / 8$. Since $G^{g}\left[V_{i} \cap W_{j}\right]$ has minimum degree at least $n / 8-\left\lceil 3 \eta^{4} N\right\rceil>4$, we deduce that it contains an $(u, v)$-path $P$ of length 3 for some $u, v \in V_{i} \cap W_{j}$. Let $P^{-}=P \backslash\{u, v\}$.

By Lemma 11, there exists a green $(u, v)$-path $P^{\prime}$ of length $2 \min \left\{|S|-\left|P^{-}\right|-\right.$ $\left.2\left\lceil 3 \eta^{4} N\right\rceil,|Q|\right\}$ in $G^{g}\left[Q, S \backslash P^{-}\right]$. We have $|Q|=\tau n \geq(1 / 2+\eta) n$ and $|S|-\left|P^{-}\right|-$ $2\left\lceil 3 \eta^{4} N\right\rceil>\sigma n-7 \eta^{4} N>(1 / 2+\eta / 2) n$. Hence, $P \cup P^{\prime}$ form a green odd cycle longer than $(1+\eta / 100) n$.

\section{Regularity Lemma for graphs}

The Szemerédi Regularity Lemma [24] asserts that each graph of positive edgedensity can be approximated by a union of a bounded number of random-like bipartite graphs. To formulate it, we need the concept of $\varepsilon$-regular pairs.

Definition 23. Let $G=(V, E)$ be a graph and let $0<\varepsilon \leq 1$. A pair $(A, B)$ of two disjoint subsets of $V$ is called $\varepsilon$-regular (with respect to $G$ ) if

$$
\left|d\left(A^{\prime}, B^{\prime}\right)-d(A, B)\right|<\varepsilon
$$

for any two subsets $A^{\prime} \subset A, B^{\prime} \subset B$ satisfying $\left|A^{\prime}\right| \geq \varepsilon|A|,\left|B^{\prime}\right| \geq \varepsilon|B|$. 
This definition states that the edges are uniformly distributed in a regular pair. The Regularity Lemma of Szemerédi [24] asserts that we can partition the vertex set $V(G)$ of a graph $G$ into $\nu$ sets $V_{1} \cup \ldots \cup V_{\nu}$ so that most of the pairs $\left(V_{i}, V_{j}\right)$ satisfy Definition 23. The precise statement, extended to edge-colored graphs, is as follows.

Theorem 24 (Multicolor Regularity Lemma). For every $\varepsilon>0$ and $k, \kappa \in \mathbb{N}$ there exist two integers $N_{0}=N_{0}(\varepsilon, k, \kappa)$ and $T_{0}:=T_{0}(\varepsilon, k, \kappa)$ with the following property: if we color the edges of any graph $G$ on $N \geq N_{0}$ vertices with $k$ colors and denote by $G^{1}, \ldots, G^{k}$ the subgraphs defined by these colors, then there is a partition of $V(G)$ into $\nu$ classes

$$
V(G)=V_{1} \cup \ldots \cup V_{\nu}
$$

such that

(A) $\kappa \leq t \leq T_{0}$,

(B) $\left|V_{i}\right| \geq\lfloor N / \nu\rfloor$ for every $i, 1 \leq i \leq \nu$, and

(C) all but at most $\varepsilon\left(\begin{array}{c}\nu \\ 2\end{array}\right)$ pairs $\left(V_{i}, V_{j}\right), 1 \leq i<j \leq \nu$, are $\varepsilon$-regular with respect to every $G^{i}, 1 \leq i \leq k$.

Remark 25. The original Regularity Lemma refers to the case $k=1$. The proof is (basically) the same for an arbitrary fixed number $k$ of colors. This version was used, for example, in [6], and formulated in the survey [17].

In our proof we also use some simple properties of $\varepsilon$-regular pairs. One of them is that they contain paths of any prescribed length between almost all pairs of vertices provided that some natural parity condition is satisfied (see also [20]).

Claim 26. Suppose $0<\varepsilon<1 / 100$. Let $\left(V_{1}, V_{2}\right)$ be an $\varepsilon$-regular pair in a graph $G$ and $d\left(V_{1}, V_{2}\right)>\varepsilon^{1 / 4}$. Then, for all but at most $\varepsilon\left|V_{1}\right|$ vertices $v_{1} \in V_{1}$, for all but at most $\varepsilon\left|V_{2}\right|$ vertices $v_{2} \in V_{2}$, and for every $\ell, 2 \leq \ell \leq(1-2 \sqrt{\varepsilon}) \min \left\{\left|V_{1}\right|,\left|V_{2}\right|\right\}$, the bipartite graph $G\left[V_{1}, V_{2}\right]$ contains a $\left(v_{1}, v_{2}\right)$-path of length $2 \ell-1$.

This implies the following:

Claim 27. Suppose that $0<\varepsilon<1 / 100$. Let $V_{1}, V_{2}, \ldots, V_{2 r+1}$ be disjoint subsets of vertices of a graph $G$ such that $\left|V_{i}\right| \geq m$, the pair $\left(V_{i}, V_{i+1}\right)$ is $\varepsilon$-regular, and $d_{G}\left(V_{i}, V_{i+1}\right)>\varepsilon^{1 / 4}$ for each $i \in[2 r+1]$. (Take $V_{2 r+2}:=V_{1}$.) Then,

(A) for every $\ell, r \leq \ell \leq(1-5 \sqrt{\varepsilon}) r m, G$ contains a cycle of length $2 \ell+1$;

(B) for all but at most $\varepsilon\left|V_{1}\right|$ vertices $u_{1}, v_{1} \in V_{1}$, and for every $\ell, 2 r \leq \ell \leq(1-$ $5 \sqrt{\varepsilon}) \mathrm{rm}, \mathrm{G}$ contains a $\left(u_{1}, v_{1}\right)$-path of length $\ell$. 
Claim 28. Suppose $0<\varepsilon<1 / 100$. Let $V_{1}, V_{2}, \ldots, V_{2 r}$ be disjoint subsets of vertices of a graph $G$ such that $\left|V_{i}\right| \geq m$, the pair $\left(V_{i}, V_{i+1}\right)$ is $\varepsilon$-regular, and $d_{G}\left(V_{i}, V_{i+1}\right)>$ $\varepsilon^{1 / 4}$ for each $i \in[2 r-1]$. Then, for all but at most $\varepsilon\left|V_{1}\right|$ vertices $v_{1} \in V_{1}$, for all but at most $\varepsilon\left|V_{2 r}\right|$ vertices $v_{2 r} \in V_{2 r}$, and for every $\ell, r<\ell \leq(1-5 \sqrt{\varepsilon}) r m$, G contains $a\left(v_{1}, v_{2 r}\right)$-path of length $2 \ell$.

\section{Proof of Theorem 3: Getting the exact result}

Proof. First we define all the constants. We set $\eta=1 / 2000$ and define $\varepsilon:=\eta^{64}$,

$$
c_{1}:=\eta^{16} \text { and } c_{2}:=\varepsilon=\eta^{64} .
$$

Since $(2 \eta)^{2}<10^{-5}=\eta_{6}$, Theorem 6 gives $n_{6}:=n_{6}\left((2 \eta)^{2}\right)$. The Multicolor Regularity Lemma (Theorem 24) used with $\kappa:=\max \left\{2 n_{6}, \frac{1}{\varepsilon}\right\}$ yields constants $T_{0}=T_{0}(\varepsilon, 3, \kappa)$ and $n_{24}=n_{24}(\varepsilon, 3, \kappa)$. Finally, we let

$$
N_{0}:=\max \left\{2 T_{0}, n_{24}\right\}
$$

Let $n_{1}, n_{2}, n_{3}>N_{0}$ be odd, $n=\max \left\{n_{1}, n_{2}, n_{3}\right\}$, and $N$ be an integer satisfying

$$
N>\left(4-c_{1}\right) n \text {. }
$$

Suppose a graph $G_{N}$ with $\delta\left(G_{N}\right) \geq N-c_{2} n$ is colored with 3 colors, red, blue, green, without red $C_{n_{1}}$, blue $C_{n_{2}}$, and green $C_{n_{3}}$. Denote the corresponding graphs - defined by the colors - by $G_{N}^{r}, G_{N}^{b}$ and $G_{N}^{g}$. Without loss of generality we may assume that

$$
N<\left(4+c_{1}\right) n \text {. }
$$

Now we apply Theorem 24 (the Multicolor Regularity Lemma) to $G_{N}^{r}, G_{N}^{b}$ and $G_{N}^{g}$ and obtain a partition $V_{1} \cup \ldots \cup V_{\nu}$ of $V:=V\left(G_{N}\right)$, where $\kappa \leq \nu \leq T_{0}$, that is $\varepsilon$-regular with respect to each of $G_{N}^{r}, G_{N}^{b}$ and $G_{N}^{g}$ and each $V_{i}$ satisfies $\left|V_{i}\right| \geq m:=\lfloor N / \nu\rfloor$.

We define the 3-colored cluster-graph $H_{\nu}$ on $\{1, \ldots, \nu\}$, by joining the vertices $i$ and $j$ in color $c$ if the pair $\left(V_{i}, V_{j}\right)$ is $\varepsilon$-regular, it has density at least $3 \varepsilon^{1 / 4}$ and color $c$ is used the most. (Ties are decided arbitrarily.) The partition is $\varepsilon$-regular for each of $G_{N}^{r}, G_{N}^{b}$ and $G_{N}^{g}$, therefore there are at most $3 \varepsilon\left(\begin{array}{c}\nu \\ 2\end{array}\right)$ irregular pairs. Since $\delta\left(G_{N}\right) \geq N-c_{2} n$ and each pair with density smaller than $3 \varepsilon^{1 / 4} \leq 1 / 4$ contains at 
least $3(N / \nu)^{2} / 4$ non-edges, there are at most $4 c_{2} n N /\left(3 N^{2} / \nu^{2}\right)<\varepsilon\left(\begin{array}{l}\nu \\ 2\end{array}\right)$ pairs with density smaller than $3 \varepsilon^{1 / 4}$. Hence

$$
e\left(H_{\nu}\right) \geq(1-4 \varepsilon)\left(\begin{array}{l}
\nu \\
2
\end{array}\right)
$$

By removing at most $2 \sqrt{\varepsilon} \nu$ vertices whose degree is less than $(1-3 \sqrt{\varepsilon}) \nu$, we get a subgraph $H^{\prime}$ of $H_{\nu}$ with

$$
\nu^{\prime} \geq(1-3 \sqrt{\varepsilon}) \nu
$$

vertices and minimum degree

$$
\delta\left(H^{\prime}\right) \geq(1-5 \sqrt{\varepsilon}) \nu \geq\left(1-(2 \eta)^{8}\right) \nu^{\prime} .
$$

We claim that the induced 3-coloring of $H^{\prime}$ has no monochromatic odd cycle longer than $\left(1+(2 \eta)^{2} / 100\right) \nu^{\prime} / 4$. Indeed, suppose that $i_{1}, \ldots, i_{2 r+1}$ are the vertices of a monochromatic odd cycle with $2 r+1>\left(1+(2 \eta)^{2} / 100\right) \nu^{\prime} / 4$. We may assume that it is red. Then all pairs $\left(V_{i_{j}}, V_{i_{j+1}}\right)$ are $\varepsilon$-regular with respect to $G_{N}^{r}$ and each pair has density at least $\frac{1}{3}\left(3 \varepsilon^{1 / 4}\right)$ (by the definition of $H^{\prime}$ and $H$ ). By Claim 27, $G_{N}^{r}$ contains every odd cycle of length between $2 r+1$ and $(1-5 \sqrt{\varepsilon}) 2 r\lfloor N / \nu\rfloor$. Since

$$
2 r+1 \leq \nu^{\prime} \leq \nu \leq T_{0}<N_{0} \leq n_{1}
$$

and

$$
(1-5 \sqrt{\varepsilon}) 2 r\left\lfloor\frac{N}{\nu}\right\rfloor>\left(1-6 \sqrt{\eta^{64}}\right)\left(1+\frac{(2 \eta)^{2}}{100}\right) \frac{(1-3 \sqrt{\varepsilon}) \nu}{4} \cdot \frac{\left(4-c_{1}\right) n}{\nu}>n \geq n_{1},
$$

there exists a red $C_{n_{1}}$ in $K_{N}$ - contradicting our assumption.

Since $\nu^{\prime}$ satisfies $\nu^{\prime} \geq(1-3 \sqrt{\varepsilon}) \kappa \geq(1-3 \sqrt{\varepsilon}) 2 n_{6} \geq n_{6}$, we can apply Theorem 6 to $H^{\prime}$ and obtain that there exists a set $W \subset V\left(H^{\prime}\right) \subset V(H)=\{1, \ldots, \nu\}$ of size $4 w$, where

$$
w:=\left\lceil\left(\frac{1}{2}+\frac{(2 \eta)^{2}}{4}\right) \frac{\nu^{\prime}}{4}\right\rceil
$$

such that the induced 3-coloring of $H^{\prime}[W]$ is embeddable into either $\mathrm{EC}_{1}(w)$ or into $\mathrm{EC}_{2}(w)$. We may assume, by permuting the names of the colors if necessary, that the induced 3-coloring of $H^{\prime}[W]$ is actually $\mathrm{EC}_{1}(w)$ or $\mathrm{EC}_{2}(w)$.

We will deal only with the harder case, when the coloring is $\operatorname{EC}_{1}(w)$. The case when the coloring is $\mathrm{EC}_{1}(w)$ follows essentially the same proof line. Hence, suppose that $W$ splits into 4 sets $W_{1}, \ldots, W_{4}$, each of size $w$, so that there is an injection 
$f: W_{i} \rightarrow X_{i}, i=1,2,3,4$, such that for every edge $x y$ in $H^{\prime}[W], x y$ has the same color in $H^{\prime}$ as $f(x) f(y)$ in $\mathrm{EC}_{1}(w)$. Let $U_{i}^{*}:=\bigcup_{V_{j} \in W_{i}} V_{j}, i=1,2,3,4$, and $U^{*}:=U_{1}^{*} \cup U_{2}^{*} \cup U_{3}^{*} \cup U_{4}^{*}$. Now we show that $G_{N}\left[U^{*}\right]$ has a large and dense subgraph whose coloring is $\mathrm{EC}_{1}\left(\left\lceil\left(1 / 2+\eta^{2}\right) n\right\rceil\right)$.

First, we delete from $G_{N}\left[U^{*}\right]$ all the edges in the pairs corresponding to the edges in the complement $\bar{H}^{\prime}$ of $H^{\prime}$. Since $e\left(\bar{H}^{\prime}\right) \leq e(\bar{H}) \leq 4 \varepsilon\left(\begin{array}{c}\nu \\ 2\end{array}\right)$, we remove this way at $\operatorname{most} 4 \varepsilon\left(\begin{array}{c}\nu \\ 2\end{array}\right) \cdot(m+1)^{2} \leq 3 \eta^{64} N^{2}$ edges.

Secondly, we delete from $K_{N}\left[U^{*}\right]$ all the edges within clusters $V_{j}$, where $j \in$ $W_{1} \cup W_{2} \cup W_{3} \cup W_{4}$, discarding at most $\nu \cdot(N / \nu)^{2} \leq \varepsilon N^{2}=\eta^{64} N^{2}$ edges.

We also remove at most $\left(\begin{array}{l}\nu \\ 2\end{array}\right) \cdot 3 \varepsilon^{1 / 4}(m+1)^{2} \leq 2 \eta^{16} N^{2}$ edges from the pairs with density smaller than $3 \varepsilon^{1 / 4}$. Finally, we delete one more set of edges, but we need to state the following claim first.

Claim 29. There exists a permutation $\left(\alpha_{1}, \alpha_{2}, \alpha_{3}, \alpha_{4}\right)$ of $(1,2,3,4)$ for which all of the following hold:

(a) For every $j \in\{1,2,3,4\}$ and for every $i, i^{\prime} \in W_{\alpha_{j}}, i \neq i^{\prime}$, we have $e_{r}\left(V_{i}, V_{i^{\prime}}\right) \leq$ $\varepsilon^{1 / 4}(m+1)^{2}$ and $e_{b}\left(V_{i}, V_{i^{\prime}}\right) \leq \varepsilon^{1 / 4}(m+1)^{2}$.

(b) Suppose that $i \in W_{\alpha_{j}}, i^{\prime} \in W_{\alpha_{j^{\prime}}}$ for some $1 \leq j<j^{\prime} \leq 4$. Then $e_{g}\left(V_{i}, V_{i^{\prime}}\right) \leq$ $\varepsilon^{1 / 4}(m+1)^{2}$.

(c) Let $i \in W_{\alpha_{j}}, i^{\prime} \in W_{\alpha_{j^{\prime}}}$, where $\left(j, j^{\prime}\right)=(1,3)$ or $(2,4)$. Then $e_{b}\left(V_{i}, V_{i^{\prime}}\right) \leq$ $\varepsilon^{1 / 4}(m+1)^{2}$.

(d) Let $i \in W_{\alpha_{j}}, i^{\prime} \in W_{\alpha_{j^{\prime}}}$, where $\left(j, j^{\prime}\right)=(1,2)$ or $(3,4)$. Then $e_{r}\left(V_{i}, V_{i^{\prime}}\right) \leq$ $\varepsilon^{1 / 4}(m+1)^{2}$.

The proof of Claim 29 appears at the end of this section. We remove all the red edges given by (a) and (d) from the pairs $\left(V_{i}, V_{i^{\prime}}\right)$, all the blue edges given by (a) and (c) from the pairs $\left(V_{i}, V_{i^{\prime}}\right)$, and all the green edges given by (b) from the pairs $\left(V_{i}, V_{i^{\prime}}\right)$. It follows from Claim 29 that we deleted at most

$$
3 \cdot\left(\begin{array}{l}
\nu \\
2
\end{array}\right) \cdot \varepsilon^{1 / 4}(m+1)^{2} \leq 2 \eta^{16} N^{2}
$$

edges. Thus we obtain a subgraph $G^{\prime} \subset G_{N}\left[U^{*}\right]$ such that

$$
e\left(G_{N}\left[U^{*}\right] \backslash G^{\prime}\right) \leq 3 \eta^{64} N^{2}+\eta^{64} N^{2}+2 \eta^{16} N^{2}+2 \eta^{16} N^{2} \leq 5 \eta^{16} N^{2} .
$$

Each set $U_{i}^{*}$ has at least

$$
\left|W_{i}\right| \cdot m \geq w\left\lfloor\frac{N}{\nu}\right\rfloor \geq \frac{\left(1+2 \eta^{2}\right)(1-3 \sqrt{\varepsilon}) \nu}{8} \cdot \frac{\left(4-c_{1}\right) n}{\nu} \geq\left(\frac{1}{2}+\frac{3 \eta^{2}}{2}\right) n
$$


vertices. We remove all the vertices of $U^{*}$ that are adjacent to at most $\left|U^{*}\right|-\left\lceil 4 \eta^{4} N\right\rceil$ vertices of $U^{*}$. Since $e\left(G_{N}\left[U^{*}\right] \backslash G^{\prime}\right) \leq 5 \eta^{16} N^{2}$, we remove at most $3 \eta^{4} N$ vertices. By removing a few further vertices (if needed), we obtain the sets $U_{i} \subset U_{\alpha_{i}}^{*}, i=1,2,3,4$, of the same size such that for $t:=\left\lceil 4 \eta^{4} N\right\rceil$, we have

(i) $\left|U_{i}\right|=\frac{1}{2}(n+13)+2 t \stackrel{(13)}{\leq}\left(1 / 2+3 \eta^{2} / 2\right) n-3 \eta^{4} N$;

(ii) $G^{\prime}\left[U_{1} \cup U_{2} \cup U_{3} \cup U_{4}\right]$ is $t$-complete;

(iii) the induced 3-coloring of $G^{\prime}\left[U_{1} \cup U_{2} \cup U_{3} \cup U_{4}\right]$ is $\operatorname{EC}_{1}\left(\frac{1}{2}(n+13)+2 t\right)$.

Applying Theorem 5, we obtain Theorem 3. What remains to prove is Claim 29.

\section{Proof.}

Part (a): Let $i, i^{\prime} \in W_{1}$. Since $H^{\prime}$ is $\lceil 5 \sqrt{\varepsilon} \nu\rceil$-complete, by Lemma 11 , there is a blue $\left(i, i^{\prime}\right)$-path of length $2(w-2\lceil 5 \sqrt{\varepsilon} \nu\rceil)$ in $H^{\prime}\left[W_{1}, W_{2}\right]$. By Claim 28 , all but at most $2 \varepsilon(m+1)^{2}$ pairs of vertices $v_{i} v_{i^{\prime}}, v_{i} \in V_{i}, v_{i^{\prime}} \in V_{i^{\prime}}$ are joined by a blue path of any prescribed length $2 \ell$ satisfying

$$
2(w-2\lceil 5 \sqrt{\varepsilon} \nu\rceil)<2 \ell \leq 2(1-5 \sqrt{\varepsilon})(w-2\lceil 5 \sqrt{\varepsilon} \nu\rceil) m .
$$

For $2 \ell=n_{2}-1$, we have

$$
2(w-2\lceil 5 \sqrt{\varepsilon} \nu\rceil) \leq \frac{\left(1+2 \eta^{2}\right) \nu^{\prime}}{4} \leq \nu \leq T_{0}<n-1 \leq n_{2}-1=2 \ell
$$

and

$$
\begin{aligned}
2(1-5 \sqrt{\varepsilon})(w-2\lceil 5 \sqrt{\varepsilon} \nu\rceil) m \\
\quad \geq(1-5 \sqrt{\varepsilon})\left(\frac{\left(1+2 \eta^{2}\right) \nu^{\prime}}{4}-4\lceil 5 \sqrt{\varepsilon} \nu\rceil\right)\left\lfloor\frac{N}{\nu}\right\rfloor \\
\quad \geq(1-6 \sqrt{\varepsilon})\left(\frac{\left(1+2 \eta^{2}\right)(1-3 \sqrt{\varepsilon}) \nu}{4}-4\lceil 5 \sqrt{\varepsilon} \nu\rceil\right) \frac{\left(4-c_{1}\right) n}{\nu} \\
\quad \geq\left(1-6 \sqrt{\eta^{64}}\right)\left(\frac{1+2 \eta^{2}}{4}-21 \sqrt{\eta^{64}}\right)\left(4-\eta^{16}\right) n \\
>n \geq n_{2}-1=2 \ell .
\end{aligned}
$$

Hence, since $G_{n}$ does not contain a blue $C_{n_{2}}$, none of these pairs $v_{i} v_{i^{\prime}}$ can be blue either.

There is also a red $\left(i, i^{\prime}\right)$-path of length $2(w-2\lceil 5 \sqrt{\varepsilon} \nu\rceil)$ in $H^{\prime}\left[W_{1}, W_{3}\right]$ and, the same argument as above yields that there are at most $2 \varepsilon(m+1)^{2}<\varepsilon^{1 / 4}(m+1)^{2}$ red edges in $\left(V_{i}, V_{i^{\prime}}\right)$. We obtain the same conclusion for $i, i^{\prime} \in W_{j}, j=2,3,4$. 
Part (b): Let $i \in W_{1}$ and $i^{\prime} \in W_{2}$ be arbitrary. Since $H^{\prime}$ is $\lceil 5 \sqrt{\varepsilon} \nu\rceil$-complete and $w>2\lceil 5 \sqrt{\varepsilon} \nu\rceil+4$, by Lemma 10, there is a green odd cycle containing $i$ of length at least $w-1$ in $H^{\prime}\left[W_{1}\right]$. Similarly, there is a green odd cycle containing $v i^{\prime}$ of length at least $w-1$ in $H^{\prime}\left[W_{2}\right]$.

Consequently, by Claim 27(B), all but at most $2 \varepsilon(m+1)^{2}$ pairs of vertices in $V_{i}$ $\left(V_{i^{\prime}}\right.$, respectively) are joined by a green path of any prescribed length between $w-1$ and $(1-5 \sqrt{\varepsilon})(w-1) m$. Notice that

$$
w-1 \leq \frac{\left(1+2 \eta^{2}\right) \nu^{\prime}}{4} \leq \frac{\nu}{2} \leq \frac{T_{0}}{2}<\frac{n-1}{2} \leq \frac{n_{3}-1}{2}
$$

and

$$
(1-5 \sqrt{\varepsilon})(w-1) m \geq(1-5 \sqrt{\varepsilon})(w-2\lceil 5 \sqrt{\varepsilon} \nu\rceil) m \geq \frac{n-1}{2} \geq \frac{n_{3}-1}{2}
$$

(see the calculation in part (a)).

If the pair $\left(V_{i}, V_{i^{\prime}}\right)$ contained more than $\varepsilon^{1 / 4}(m+1)^{2}$ green edges, we could find two green independent edges $u_{i} u_{i^{\prime}}$ and $v_{i} v_{i^{\prime}}, u_{i}, v_{i} \in V_{i}, u_{i^{\prime}}, v_{i^{\prime}} \in V_{i^{\prime}}$, such that $u_{i}, v_{i}$ and $u_{i^{\prime}}, v_{i^{\prime}}$ can be joined by disjoint green paths of any length between $w-1$ and $(1-5 \sqrt{\varepsilon})(w-1) m$. Hence, we could find an odd green $\left(u_{i}, v_{i}\right)$-path $P$ and an even green $\left(u_{i^{\prime}}, v_{i^{\prime}}\right)$-path $P$ such that

$$
|P|+\left|P^{\prime}\right|=n_{3}-2 .
$$

This is a contradiction because $P \cup P^{\prime} \cup\left\{u_{i} u_{i^{\prime}}, v_{i} v_{i^{\prime}}\right\}$ is a green $C_{n_{3}}$. Therefore, $e_{g}\left(V_{i}, V_{i^{\prime}}\right) \leq \varepsilon^{1 / 4}(m+1)^{2}$.

Part (c): If $e_{b}\left(V_{i}, V_{i^{\prime}}\right) \leq \varepsilon^{1 / 4}(m+1)^{2}$ for all $i \in W_{j}, i^{\prime} \in W_{j^{\prime}}$ and for $\left(j, j^{\prime}\right)=$ $(1,3),(2,4)$, then we set $\alpha_{i}=i$ for $i=1,2,3,4$.

Hence, without loss of generality, suppose that $1 \in W_{1}, 3 \in W_{3},\left(V_{1}, V_{3}\right)$ is $\varepsilon$-regular w.r.t. $G_{N}^{b}$, and $e_{b}\left(V_{1}, V_{3}\right)>\varepsilon^{1 / 4}(m+1)^{2}$. Now we show that

$$
\begin{gathered}
e_{b}\left(V_{i}, V_{i^{\prime}}\right) \leq \varepsilon^{1 / 4}(m+1)^{2} \quad \text { for all } i \in W_{j}, i^{\prime} \in W_{j^{\prime}}, i i^{\prime} \in H^{\prime}, \text { and } \\
\left(j, j^{\prime}\right)=(1,4),(2,3) .
\end{gathered}
$$

Indeed, suppose that $i \in W_{1}, i^{\prime} \in W_{4}$ are such that $i i^{\prime} \in H^{\prime}$ and $e_{b}\left(V_{i}, V_{i^{\prime}}\right)>$ $\varepsilon^{1 / 4}(m+1)^{2}$. By Lemma 11, there is a $(1, i)$-path $P$ of length 2 (or 0 if $i=1$ ) in the blue $\lceil 5 \sqrt{\varepsilon} \nu\rceil$-complete graph $H^{\prime}\left[W_{1}, W_{2}\right]$. Since $H^{\prime}\left[W_{3}, W_{4}\right]$ is also blue and 
$\lceil 5 \sqrt{\varepsilon} \nu\rceil$-complete, the same lemma implies the existence of a blue odd $\left(i^{\prime}, 3\right)$-path $P^{\prime}$ of length $2 w-4\lceil 5 \sqrt{\varepsilon} \nu\rceil-1$ in $H^{\prime}\left[W_{3}, W_{4}\right]$. The edges 13 , $i i^{\prime}$ and paths $P, P^{\prime}$ form an odd cycle of length at least $2 w-4\lceil 5 \sqrt{\varepsilon} \nu\rceil+1$. Every two consecutive clusters in this cycle form an $\varepsilon$-regular pair with the blue edge density at least $\varepsilon^{1 / 4}$. Claim 27 implies that $G$ contains a blue cycle of length $2 \ell+1$ provided

$$
2(w-2\lceil 5 \sqrt{\varepsilon} \nu\rceil)+1 \leq 2 \ell+1 \leq 2(1-5 \sqrt{\varepsilon})(w-2\lceil 5 \sqrt{\varepsilon} \nu\rceil) m+1 .
$$

From part (a), we have

$$
2(w-2\lceil 5 \sqrt{\varepsilon} \nu\rceil)+1 \leq n_{2} \leq 2(1-5 \sqrt{\varepsilon})(w-2\lceil 5 \sqrt{\varepsilon} \nu\rceil) m+1,
$$

hence $G$ contains a blue $C_{n_{2}}$, which is a contradiction. We obtain a contradiction in the same way for $i \in W_{2}, i^{\prime} \in W_{3}$.

From (14) it follows that $\alpha_{1}=1, \alpha_{2}=2, \alpha_{3}=4, \alpha_{4}=3$ is a permutation satisfying (a)-(c).

Part (d): If $e_{r}\left(V_{i}, V_{i^{\prime}}\right) \leq \varepsilon^{1 / 4}(m+1)^{2}$ for all $i \in W_{\alpha_{j}}, i^{\prime} \in W_{\alpha_{j^{\prime}}}$ and $\left(j, j^{\prime}\right)=$ $(1,2),(3,4)$, then the proof is finished. Otherwise, there exist $i \in W_{\alpha_{j}}$ and $i^{\prime} \in W_{\alpha_{j^{\prime}}}$, where $\left(j, j^{\prime}\right)=(1,2)$ or $\left(j, j^{\prime}\right)=(3,4)$, such that $\left(V_{i}, V_{i^{\prime}}\right)$ is $\varepsilon$-regular w.r.t. $G_{N}^{r}$ and $e_{r}\left(V_{i}, V_{i^{\prime}}\right)>\varepsilon^{1 / 4}(m+1)^{2}$. Then the following holds:

$$
\begin{aligned}
& e_{r}\left(V_{i}, V_{i^{\prime}}\right) \leq \varepsilon^{1 / 4}(m+1)^{2} \text { for all } i \in W_{\alpha_{j}}, i^{\prime} \in W_{\alpha_{j^{\prime}}}, i i^{\prime} \in H^{\prime} \text {, and } \\
& \left(j, j^{\prime}\right)=(1,4),(2,3) \text {. }
\end{aligned}
$$

The proof of this statement is analogous to the proof of (14) and we omit it here. By replacing $\alpha_{2}$ with $\alpha_{4}$ and $\alpha_{4}$ with $\alpha_{2}$, we obtain a permutation $\left(\alpha_{1}, \alpha_{2}, \alpha_{3}, \alpha_{4}\right)$ of $(1,2,3,4)$ satisfying $(\mathrm{a})-(\mathrm{d})$.

\section{Concluding Remarks}

As we know, the behavior of Ramsey numbers for even cycles differs from that for odd ones, even for two colors (see (1)). For three colors, Figaj and Luczak [9] proved that if $n_{1} \geq n_{2} \geq n_{3}$ are even, then

$$
R\left(C_{n_{1}}, C_{n_{2}}, C_{n_{3}}\right)=n_{1}+\frac{n_{2}+n_{3}}{2}+o\left(n_{1}\right)
$$


as $n_{3} \rightarrow \infty$. This trivially implies that

$$
R\left(P_{n_{1}}, P_{n_{2}}, P_{n_{3}}\right)=n_{1}+\frac{n_{2}+n_{3}}{2}+o\left(n_{1}\right),
$$

in particular,

$$
R\left(P_{n}, P_{n}, P_{n}\right)=2 n+o(n),
$$

where $P_{n}$ is a path on $n$ vertices.

Slightly later, independently, Gyárfás, Ruszinkó, G. Sárközy, and Szemerédi [13] proved the same result for the diagonal case (i.e. when $n_{1}=n_{2}=n_{3}$ ) and, moreover, for $n$ large, they obtained the exact result for paths of the same length:

$$
R\left(P_{n}, P_{n}, P_{n}\right)= \begin{cases}2 n-2, & n \text { is even, } \\ 2 n-1, & n \text { is odd }\end{cases}
$$

Recently, Benevides and Skokan [2] proved that $R\left(C_{n}, C_{n}, C_{n}\right)=2 n$ for all even, sufficiently large values of $n$.

We also remark that the conjecture of Bondy and Erdős extends to arbitrary number of colors:

$$
R(\underbrace{C_{n}, \ldots, C_{n}}_{k})= \begin{cases}2^{k-1}(n-1)+1, & n \text { is odd }, \\ \frac{(k+1) n}{2}+O(1), & n \text { is even },\end{cases}
$$

and it is still open for $k>3$.

\section{Acknowledgement}

A large part of this work was done at Instituto de Matemática e Estatística, Universidade de São Paulo and Rényi Institute of Mathematics, whose hospitality the authors gratefully acknowledge.

\section{References}

[1] L. Babai, M. Simonovits, and J. Spencer, Extremal subgraphs of random graphs, J. Graph Theory 14 (1990), no. 5, 599-622.

[2] F. Benevides and J. Skokan, The exact 3-colored Ramsey number for long even cycles, submitted. 
[3] J. A. Bondy, Pancyclic graphs. I. J. Combinatorial Theory Ser. B 11 (1971), 80-84.

[4] J. A. Bondy and P. Erdős, Ramsey numbers for cycles in graphs, J. Combinatorial Theory Ser. B 14 (1973), 46-54.

[5] P. Erdős and T. Gallai, On maximal paths and circuits of graphs, Acta Math. Acad. Sci. Hungar. 10 (1959), 337-356 (unbound insert).

[6] P. Erdős, A. Hajnal, V. T. Sós, and E. Szemerédi, More results on Ramsey-Turán type problems, Combinatorica 3 (1983), no. 1, 69-81.

[7] P. Erdős and M. Simonovits, An extremal graph problem, Acta Math. Acad. Sci. Hungar. 22 (1971/72), 275-282.

[8] R. J. Faudree and R. H. Schelp, All Ramsey numbers for cycles in graphs, Discrete Math. 8 (1974), 313-329.

[9] A. Figaj and T. Euczak, The Ramsey number for a triple of long even cycles, J. Combinatorial Theory Ser. B 97 (2007), no. 4, 584-596.

[10] Z. Füredi, O. Pikhurko, and M. Simonovits, The Turán density of the hypergraph $\{a b c, a d e, b d e, c d e\}$, Electron. J. Combin. 10 (2003), Research Paper 18, 7 pp. (electronic).

[11] Z. Füredi, O. Pikhurko, and M. Simonovits, On triple systems with independent neighbourhoods, Combin. Probab. Comput. 14 (2005), no. 5-6, 795-813.

[12] Z. Füredi and M. Simonovits, Triple systems not containing a Fano configuration, Combin. Probab. Comput. 14 (2005), no. 4, 467-484.

[13] A. Gyárfás, M. Ruszinkó, G. N. Sárközi, and E. Szemerédi, Three-color Ramsey number for paths, Combinatorica 27 (2007), no. 1, 35-69.

[14] P. Keevash and B. Sudakov, The Turán number of the Fano plane, Combinatorica 25 (2005), no. 5, 561-574.

[15] Y. Kohayakawa, M. Simonovits, and J. Skokan, Stability of Ramsey numbers for cycles, in preparation.

[16] Y. Kohayakawa, M. Simonovits, and J. Skokan, The 3-colored Ramsey number of odd cycles, Proceedings of GRACO2005, pp. 397-402 (electronic), Electron. Notes Discrete Math., 19, Elsevier, Amsterdam, 2005.

[17] J. Komlós and M. Simonovits, Szemerédi's regularity lemma and its applications in graph theory, Combinatorics, Paul Erdős is eighty, Vol. 2 (Keszthely, 1993), Bolyai Soc. Math. Stud., vol. 2, János Bolyai Math. Soc., Budapest, 1996, pp. 295-352.

[18] L. Lovász and M. Simonovits, On the number of complete subgraphs of a graph, Proceedings of the Fifth British Combinatorial Conference (Univ. Aberdeen, Aberdeen, 1975) (Winnipeg, Man.), Utilitas Math., 1976, pp. 431-441. Congressus Numerantium, No. XV.

[19] L. Lovász and M. Simonovits, On the number of complete subgraphs of a graph. II, Studies in pure mathematics, Birkhäuser, Basel, 1983, pp. 459-495.

[20] T. Łuczak, $R\left(C_{n}, C_{n}, C_{n}\right) \leq(4+o(1)) n$, J. Combin. Theory Ser. B 75 (1999), no. 2, 174-187. 
[21] V. Nikiforov and R. H. Schelp, Cycles and stability, J. Combin. Theory, Ser. B 98 (2008), 69-84.

[22] V. Rosta, On a Ramsey-type problem of J. A. Bondy and P. Erdös. I, II, J. Combinatorial Theory Ser. B 15 (1973), 94-104; ibid. 15 (1973), 105-120.

[23] M. Simonovits, A method for solving extremal problems in graph theory, stability problems, Theory of Graphs (Proc. Colloq., Tihany, 1966), Academic Press, New York, 1968, pp. 279-319.

[24] E. Szemerédi, Regular partitions of graphs, Problèmes combinatoires et théorie des graphes (Colloq. Internat. CNRS, Univ. Orsay, Orsay, 1976), Colloq. Internat. CNRS, vol. 260, CNRS, Paris, 1978, pp. 399-401.

[25] J. E. Williamson, Panconnected graphs II, Period. Math. Hung. 8 (1977), 105-116. 